\title{
Relic Neutrinos, thermal axions and cosmology in early 2014
}

\author{
Elena Giusarma, ${ }^{1}$ Eleonora Di Valentino, ${ }^{1}$ Massimiliano Lattanzi, ${ }^{2}$ Alessandro Melchiorri, ${ }^{1}$ and Olga Mena ${ }^{3}$ \\ ${ }^{1}$ Physics Department and INFN, Università di Roma "La Sapienza", Ple Aldo Moro 2, 00185, Rome, Italy \\ ${ }^{2}$ Dipartimento di Fisica e Science della Terra, Università di Ferrara and INFN, \\ sezione di Ferrara, Polo Scientifico e Tecnologico - Edficio C Via Saragat, 1, I-44122 Ferrara Italy \\ ${ }^{3}$ IFIC, Universidad de Valencia-CSIC, 46071, Valencia, Spain
}

\begin{abstract}
We present up to date cosmological bounds on the sum of active neutrino masses as well as on extended cosmological scenarios with additional thermal relics, as thermal axions or sterile neutrino species. Our analyses consider all the current available cosmological data in the beginning of year 2014, including the very recent and most precise Baryon Acoustic Oscillation (BAO) measurements from the Baryon Oscillation Spectroscopic Survey. In the minimal three active neutrino scenario, we find $\sum m_{\nu}<0.22 \mathrm{eV}$ at $95 \%$ CL from the combination of CMB, BAO and Hubble Space Telescope measurements of the Hubble constant.

A non zero value for the sum of the three active neutrino masses of $\sim 0.3 \mathrm{eV}$ is significantly favoured at more than 3 standard deviations when adding the constraints on $\sigma_{8}$ and $\Omega_{m}$ from the Planck Cluster catalog on galaxy number counts. This preference for non zero thermal relic masses disappears almost completely in both the thermal axion and massive sterile neutrino schemes.

Extra light species contribute to the effective number of relativistic degrees of freedom, parameterised via $N_{\text {eff. }}$. We found that when the recent detection of B mode polarization from the BICEP2 experiment is considered, an analysis of the combined CMB data in the framework of LCDM+r models gives $N_{\text {eff }}=4.00 \pm 0.41$, suggesting the presence of an extra relativistic relic at more than 95\% c.l. from CMB-only data.
\end{abstract}

PACS numbers: 98.80.-k 95.85.Sz, 98.70.Vc, 98.80.Cq

\section{INTRODUCTION}

In standard cosmology, hot, thermal relics are identified with the three light, active neutrino flavours of the Standard Model of elementary particles. The masses of these three neutrino states have an impact in the different cosmological observables, see Refs. [1, 2] for a detailed description. Traditionally, the largest effect caused by neutrino masses on the Cosmic Microwave Background (CMB) anisotropies, is via the Early Integrated Sachs Wolfe effect (ISW). Light active neutrino species may turn non-relativistic close to the decoupling period, affecting the gravitational potentials and leaving a signature which turns out to be maximal around the first acoustic oscillation peak in the photon temperature anisotropy spectrum.

More recently, the Planck satellite CMB data [3], has opened the window to tackle the neutrino mass via gravitational lensing measurements: neutrino masses are expected to leave an imprint on the lensing potential (due to the higher expansion rate) at scales smaller than the horizon when neutrinos turn non relativistic states 4 . However, the largest effect of neutrino masses on the several cosmological observables comes from the suppression of galaxy clustering at small scales. Neutrinos, being hot thermal relics, possess large velocity dispersions. Consequently, the non-relativistic neutrino overdensities will only cluster at wavelengths larger than their free streaming scale, reducing the growth of matter density fluctuations at small scales, see e.g Refs. 5-17. Non degenerate neutrinos have different free streaming scales and in principle, with perfect measurements of the matter power spectrum, the individual values of the neutrino masses could be identified. In practice, the former is an extremely challenging task. Cosmological measurements are, for practical purposes, only sensitive to the total neutrino mass, i.e. to the sum of the three active neutrino masses.

CMB Measurements from the Planck satellite, including the lensing likelihood and low- $\ell$ polarization measurements from WMAP 9-year data 18 provide a limit on the sum of the three active neutrino masses of $\sum m_{\nu}<$ $1.11 \mathrm{eV}$ at $95 \% \mathrm{CL}$. When a prior on the Hubble constant $H_{0}$ from the Hubble Space Telescope [19] is added in the analysis, the constraint is strongly tightened, being $\sum m_{\nu}<0.21 \mathrm{eV}$ at $95 \% \mathrm{CL}$, due to the huge existing degeneracy between $H_{0}$ and $\sum m_{\nu}$, see Ref. 8. The addition of Baryon Acoustic Oscillation (BAO) measurements from the Sloan Digital Sky Survey (SDSS)-II Data Release 7 [20, 21], from the WiggleZ survey [22], from the Baryon Acoustic Spectroscopic Survey (BOSS) [23], one of the four surveys of SDSS-III 24] Data Release 9 [25] and from $6 \mathrm{dF} 26$ to Planck CMB measurements also significantly improves the neutrino mass constraints, leading to $\sum m_{\nu}<0.26 \mathrm{eV}$ at $95 \% \mathrm{CL}$ (see also the recent work of [27]).

However, the former bounds are obtained assuming that neutrinos are the only hot thermal relic component in the universe. The existence of extra hot relic components, as sterile neutrino species and/or thermal axions will change the cosmological neutrino mass constraints, see Refs. [8, 13, 15, 28, 36]. Massless, sterile neutrino-like particles, arise naturally in the context of models which contain a dark radiation sector that decouples from the 
Standard Model. A canonical example are asymmetric dark matter models, in which the extra radiation degrees of freedom are produced by the annihilations of the thermal dark matter component [37, see also Refs. 38, 39] for extended weakly-interacting massive particle models. On the other hand, extra sterile massive, light neutrino species, whose existence is not forbidden by any fundamental symmetry in nature, may help in resolving the so-called neutrino oscillation anomalies 40, 41, see also Refs. 42 46] for recent results on the preferred sterile neutrino masses and abundances considering both cosmological and neutrino oscillation constraints. Another candidate is the thermal axion [47, which constitutes the most elegant solution to the strong $\mathrm{CP}$ problem, i.e. why $\mathrm{CP}$ is a respected symmetry of Quantum Chromodynamics (QCD) despite the existence of a natural, four dimensional, Lorentz and gauge invariant operator which badly violates CP. Axions are the Pseudo- Nambu-Goldstone bosons associated to a new global $U(1)_{P Q}$ symmetry, which is spontaneously broken at an energy scale $f_{a}$. The axion mass is inversely proportional to the axion coupling constant $f_{a}$

$$
m_{a}=\frac{f_{\pi} m_{\pi}}{f_{a}} \frac{\sqrt{R}}{1+R}=0.6 \mathrm{eV} \frac{10^{7} \mathrm{GeV}}{f_{a}},
$$

where $R=0.553 \pm 0.043$ is the up-to-down quark masses ratio and $f_{\pi}=93 \mathrm{MeV}$ is the pion decay constant. Axions may be copiously produced in the early universe via thermal or non-thermal processes, providing therefore, a possible hot relic candidate in the thermal case, to be considered together with the standard relic neutrino background.

Both extra, sterile neutrino species and axions have an associated free streaming scale, reducing the growth of matter fluctuations at small scales. Indeed, it has been noticed by several authors [48, 49] that the inclusion of Planck galaxy cluster number counts data [50] in the cosmological data analyses, favours a non zero value for the sterile neutrino mass: the free streaming sterile neutrino nature will reduce the matter power at small (i.e. cluster) scales but will leave unaffected the scales probed by the CMB. A similar tendency for $\sum m_{\nu}>0$ appears, albeit to a smaller extent [48, when considering CFHTLens weak lensing constraints on the clustering matter amplitude [51].

Extra dark radiation or light species as neutrinos and axions will also contribute to the effective number of relativistic degrees of freedom $N_{\text {eff }}$, defined as

$$
\rho_{\text {rad }}=\left[1+\frac{7}{8}\left(\frac{4}{11}\right)^{4 / 3} N_{\mathrm{eff}}\right] \rho_{\gamma},
$$

where $\rho_{\gamma}$ is the present energy density of the CMB. The canonical value $N_{\text {eff }}=3.046$ corresponds to the three active neutrino contribution. If there are extra light species at the Big Bang Nucleosynthesis (BBN) epoch, the expansion rate of the universe will be higher, leading to a higher freeze out temperature for the weak interactions which translates into a higher primordial helium fraction. The most recent measurements of deuterium [52] and helium [53] light element abundances provide the constraint $N_{\text {eff }}=3.50 \pm 0.20$ [52].

It is the aim of this paper to analyse the constraints on the three active neutrino masses, extending the analyses to possible scenarios with additional hot thermal relics, as sterile neutrino species or axions, using the available cosmological data in the beginning of this year 2014 . The data combination used here includes also the recent and most precise distance BAO constraints to date from the BOSS Data Release 11 (DR11) results [54, see also Refs. [55 57.

The structure of the paper is as follows. Section II describes the different cosmological scenarios with hot thermal relics explored here and the data used in our numerical analyses. In Sec. III we present the current limits using the available cosmological data in the three active neutrino massive scenario, and in this same scheme but enlarging the hot relic component firstly with thermal axions, secondly with additional dark radiation (which could be represented, for instance, by massless sterile neutrino species) and finally, with massive sterile neutrino species. We draw our conclusions in Sec. V.

\section{COSMOLOGICAL DATA ANALYSES}

The baseline scenario we analyse here is light active massive neutrino scheme with three degenerate massive neutrinos, described by the parameters:

$$
\left\{\omega_{b}, \omega_{c}, \Theta_{s}, \tau, n_{s}, \log \left[10^{10} A_{s}\right], \sum m_{\nu}\right\},
$$

$\omega_{b} \equiv \Omega_{b} h^{2}$ and $\omega_{c} \equiv \Omega_{c} h^{2}$ being the physical baryon and cold dark matter energy densities, $\Theta_{s}$ the ratio between the sound horizon and the angular diameter distance at decoupling, $\tau$ is the reionization optical depth, $n_{s}$ the scalar spectral index, $A_{s}$ the amplitude of the primordial spectrum and $\sum m_{\nu}$ the sum of the masses of the three active neutrinos in $\mathrm{eV}$. We then consider simultaneously the presence of two hot relics, both massive neutrinos and axions, enlarging the former scenario with one thermal axion of mass $m_{a}$, see Appendix VII for details concerning the calculation of the axion energy density as a function of the cosmic time. The other possibility is the existence of extra dark radiation species, that we have firstly addressed by introducing a number of massless sterile neutrino-like species, parameterized via $N_{\text {eff }}$ (together with the baseline three massive neutrino total mass $\left.\sum m_{\nu}\right)$. The extra additional sterile states, if massive, may help in resolving the so-called neutrino oscillation anomalies. Consequently, we also constrain here simultaneously the $N_{\text {eff }}$ massive sterile neutrino scenario and the sum of the three active neutrino masses $\sum m_{\nu}$. The effective number of massive sterile neutrino species is represented by $\Delta N_{\text {eff }}=N_{\text {eff }}-3.046$, and its mass is $m_{s}^{\text {eff }}$, which is related to the physical sterile neutrino mass via 
the relation:

$$
m_{s}^{\mathrm{eff}}=\left(T_{s} / T_{\nu}\right)^{3} m_{s}=\left(\Delta N_{\mathrm{eff}}\right)^{3 / 4} m_{s},
$$

being $T_{s}\left(T_{\nu}\right)$ the current temperature of the sterile (active) neutrino, and assuming that the sterile states are hot thermal relics with a phase space distribution similar to the active neutrino one.

Table 1 specifies the priors considered on the different cosmological parameters. For our numerical analyses, we have used the Boltzmann CAMB code [58] and extracted cosmological parameters from current data using a Monte Carlo Markov Chain (MCMC) analysis based on the publicly available MCMC package cosmomc 59.

In particular, we run chains using the MetropolisHastings (MH) algorithm to obtain posterior distributions for the model parameters, given a certain dataset combination. The only exception is for the measurements of the power spectrum amplitude (described in the following section), that are included in our analysis by postprocessing the $\mathrm{MH}$ chains that were previously generated without accounting for these data. The post-processing is done using the technique of importance sampling; this technique is very reliable when the posterior distributions obtained after including new data are centered on the same values as the old distributions, and becomes on the contrary less reliable the more the new posteriors are shifted with respect to the old ones. The reason for this is that in this case one needs to sample from the low-probability tail of the old distribution, that is poorly explored by the $\mathrm{MH}$ algorithm (unless the chains run for a very long time). We stress this fact since, as we shall see in the following, the inclusion of the data on the power spectrum amplitude shifts the posterior for some of the model parameters.

All the cases under consideration (additional massless species, massive sterile neutrinos, and axions) can be studied with none or minimal to modifications to the CAMB code. In particular, the massive sterile and axion cases can be reproduced in the Boltzmann code by means of a suitable reparameterization and by treating, code-wise, the additional species as massive neutrinos. This relies on the fact that, for an equilibrium distribution function, the evolution equations only depend on the mass over temperature ratio $m_{i} / T_{i}$ and on the total density $\Omega_{i}(i=\mathrm{a}, \mathrm{s})$. The equivalence is perfect for thermal sterile neutrinos, because they have a Fermi-Dirac distribution function like ordinary neutrinos; instead, this is not the case for thermal axions since they are described by a Bose-Einstein distribution function. We take into account here the bosonic nature of axions at the background level, but not in the perturbation equations. However we argue that the error that we commit in keeping the Fermi-Dirac distribution function in the perturbation equations for axions is negligible given the uncertainties on the model parameters.

\begin{tabular}{c|c}
\hline \hline Parameter & Prior \\
\hline$\Omega_{b} h^{2}$ & $0.005 \rightarrow 0.1$ \\
$\Omega_{c} h^{2}$ & $0.01 \rightarrow 0.99$ \\
$\Theta_{s}$ & $0.5 \rightarrow 10$ \\
$\tau$ & $0.01 \rightarrow 0.8$ \\
$n_{s}$ & $0.9 \rightarrow 1.1$ \\
$\ln \left(10^{10} A_{s}\right)$ & $2.7 \rightarrow 4$ \\
$\sum m_{\nu}[\mathrm{eV}]$ & $0.06 \rightarrow 3$ \\
$m_{a}[\mathrm{eV}]$ & $0.1 \rightarrow 3$ \\
$N_{\mathrm{eff}}$ & $0(3.046) \rightarrow 10$ \\
$m_{s}^{\text {eff }}[\mathrm{eV}]$ & $0 \rightarrow 3$ \\
\hline \hline
\end{tabular}

TABLE I: Uniform priors for the cosmological parameters considered here. In the case of the extra relativistic degrees of freedom $N_{\text {eff }}$, the numbers refer to the massless (massive) case.

\section{A. Cosmological data}

\section{1. $C M B$ data}

We consider the data on CMB temperature anisotropies measured by the Planck satellite (including information on the lensing potential) 6062 combined with 9-year polarization data from WMAP [18] and with additional temperature data from highresolution CMB experiments, namely the Atacama Cosmology Telescope (ACT) 63 and the South Pole Telescope (SPT) 64.

The likelihood functions associated to these datasets are estimated and combined using the likelihood code distributed by the Planck collaboration, described in Refs. 61 and 62, and publicly available at Planck Legacy Archive $^{1}$. The Planck TT likelihood is constructed following a hybrid approach: the high- $\ell(\ell \geq 50)$ part is based on a pseudo- $C_{\ell}$ technique and uses power spectra estimated from the detectors of the 100, 143 and $217 \mathrm{GHz}$ frequency channels, while the low- $\ell(\ell \leq 49)$ part uses a Gibbs sampling-based approach and combines data from all frequencies from 30 to $353 \mathrm{GHz}$. We use Planck TT data up to a maximum multipole number of $\ell_{\max }=2500$. These are supplemented by the low- $\ell$ WMAP 9-year polarization likelihood, that includes multipoles up to $\ell=23$ 18. For what concerns the smallscale observations, we follow the approach of the Planck collaboration, as implemented in their likelihood code, and include the ACT spectra presented in Ref. 63] and the SPT spectra presented in Ref. 64. In particular, the likelihood uses the ACT $148 \times 148$ spectra in the range $1000<\ell<9440$, the ACT $148 \times 218$ and $218 \times 218$ spectra in the range $1500<\ell<9440$, and the SPT 95, 150 and $220 \mathrm{GHz}$ spectra in the range $2000<\ell<10000$, as described in Sec. 4.1 of Ref. [3]. The primary purpose of

\footnotetext{
1 http://pla.esac.esa.int/pla/aio/planckProducts.html
} 
considering these subsets of the ACT and SPT data is to improve the constraints on the unresolved foregrounds. Finally, we use the information on the gravitational lensing power spectrum estimated from the trispectrum of the Planck maps, as implemented in the Planck lensing likelihood described in Ref. 62.

We shall refer to the combination of all the abovementioned data as the $C M B$ data set.

In our analysis of the CMB dataset, we compute the helium abundance following the BBN theoretical prediction, in which the helium mass fraction is a function of $\Omega_{b} h^{2}$ and $N_{\text {eff }}$ (see the BBN section below) and fix the lensing spectrum normalization to $A_{L}=1$. We marginalize over all foregrounds parameters as described in [3].

\section{Large scale structure data}

We consider here several large scale structure data sets in different forms. First of all, we include all the available galaxy survey measurements in the form of Baryon Acoustic Oscillation (BAO) data. As a novelty, we add to the existing BAO data sets (SDSS Data Release 7 [20, 21, WiggleZ survey [22], 6dF [26]) the most recent and most accurate $\mathrm{BAO}$ measurements to date, arising from the BOSS Data Release 11 (DR11) results 54. Using approximately a sample of one million galaxies and covering 8500 squared degrees, the DR11 results provide the constraints on the spherically averaged distance $D_{V} / r_{d}{ }^{2}$ to be $13.42 \pm 0.13$ and $8.25 \pm 0.16$ at redshifts $z=0.57$ and $z=0.32$, respectively. We present results separately for DR11 BAO measurements, as well as the combination of the former results with other previous BAO measurements, referring to them as $D R 11$ and $B A O$, respectively.

We also exploit here the WiggleZ survey large scale structure measurements in their full matter power spectrum form [66, in order to quantify the benefits of using shape measurements of the matter power spectrum versus geometrical BAO information in extended cosmological scenarios with additional degeneracies among the different parameters, see the earlier work of Refs. 6, 8] where similar comparisons were performed. This data set is referred as $W Z$, and whenever it is included, the BAO measurement from the WiggleZ survey is not considered in the BAO data set.

\section{Supernova luminosity distance and Hubble constant measurements}

Supernova luminosity distance measurements from the first three years of the Supernova Legacy Survey [67] are

\footnotetext{
2 The value of the sound horizon $r_{d}$ used for these values is obtained using the Eisenstein \& Hu fitting formula 65.
}

included in the hot thermal dark matter relic bounds presented here, referring to these data as $S N L S$.

Our cosmological data analyses will also address the effect of a gaussian prior on the Hubble constant $H_{0}=$ $73.8 \pm 2.4 \mathrm{~km} / \mathrm{s} / \mathrm{Mpc}$, accordingly with the measurements from the Hubble Space Telescope [19]. We refer to this prior as $H S T$.

\section{Additional data sets: $\sigma_{8}$ measurements}

Measurements of the galaxy power shear spectra by tomographic weak lensing surveys provide a powerful tool to set constraints on the mass distribution in the universe. The amplitude and the shape of the weak lensing signal are sensitive to the normalization of the power spectrum, the so-called $\sigma_{8}$ parameter (which is the standard deviation of the matter density perturbations in a sphere of radius $8 \mathrm{Mpc} / h$ ), as well as to the overall matter energy density of the universe, $\Omega_{m}$. Using six tomographic redshift bins spanning from $z=0.28$ to $z=1.12$, the CFHTLens survey finds $\sigma_{8}\left(\Omega_{m} / 0.27\right)^{0.46}=$ $0.774_{-0.041}^{+0.032}$ [51. We shall use this constraint in our analyses, applying this constraint to our Monte Carlo Markov chains.

A strong and independent measurement of the amplitude of the power spectrum arises from the abundance of clusters as a function of the redshift, being the cluster redshift distribution a powerful probe of both $\Omega_{m}$ and $\sigma_{8}$. The Planck Sunyaev-Zeldovich (SZ) selected clusters catalog, which consists of 189 galaxy clusters with measured redshift in the $\mathrm{X}$ range, is the largest $\mathrm{SZ}$ cluster sample to date and has provided the constraint $\sigma_{8}\left(\Omega_{m} / 0.27\right)^{0.3}=0.782 \pm 0.010$ [50] via the cluster mass function. We will address as well this constraint in our Monte Carlo Markov chain analyses. These measurements are included in our analysis by post-processing the chains that were previously generated without accounting for these data.

\section{Big Bang Nucleosynthesis}

The light elements abundance is also sensitive to several cosmological parameters. The primordial abundance of deuterium is usually considered as an invaluable baryometer, since the higher the baryon abundance $\Omega_{b} h^{2}$, the less deuterium survives. On the other hand, while the mass fraction of helium- $4{ }^{4} \mathrm{He}\left(Y_{p}\right)$ is rather insensitive to $\Omega_{b} h^{2}$, it is directly related to the expansion rate at the $\mathrm{BBN}$ period, which strongly depends on the effective number of relativistic degrees of freedom

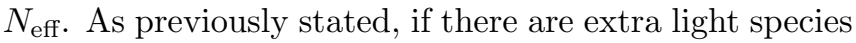
at the $\mathrm{BBN}$ epoch, the expansion rate of the universe will be higher, leading to a higher freeze out temperature for the weak interactions which translates into a higher primordial helium fraction $Y_{p}$. Here we exploit the primordial deuterium values from Ref. 68 $(D / H)_{p}=$ 
$(2.87 \pm 0.22) \times 10^{-5}$ as well as the most recent deuterium measurements $(D / H)_{p}=(2.53 \pm 0.04) \times 10^{-5}$ [52], to compare the cosmological constraints obtained with these two diferent primordial deuterium estimates, including also the measurements of the helium mass fraction $Y_{p}=0.254 \pm 0.003$ [53]. We shall use the former constraints in the scenarios in which extra relativistic degrees of freedom are expected to be present at the BBN period.

Notice that Planck CMB data is also sensitive to the value of $Y_{p}$ via measurements of the CMB damping tail (high multipole region), and therefore we use the BBN consistency option of the MCMC software exploited here, cosmomc [59], assuming therefore that the value of the extra relativistic degrees of freedom remains unchanged between the BBN and the CMB epochs. Then, given a cosmological model, the theoretical primordial abundance of helium, which is a function of $\Omega_{b} h^{2}$ and $N_{\text {eff }}{ }^{3}$ is computed, using AlterBBN [70, a numerical code devoted to calculate the BBN abundances within non standard cosmologies. We perform a similar calculation for the deuterium primordial abundance, and then fit the theoretical expectations for the deuterium and helium primordial abundances (previously computed for the CMB data analyses in the latter case) to the measurements quoted above, adding the resulting likelihood in our MCMC analyses by means of a postprocessing of our chains.

\section{Consistency of datasets}

We derive our constraints on model parameters using different combinations of the datasets described in the previous sections. However, in a few cases there are tensions between datasets, that we describe in the following. We also briefly assess, at least qualitatively, the effect on parameters of adding these data.

We use the Planck lensing likelihood in all our analyses. The lensing likelihood is based on the information encoded in the 4-point correlation function (i.e., the trispectrum) of $\mathrm{CMB}$ temperature anisotropies. On the other hand, lensing also directly affects the CMB power spectrum. As explained in Sec. 5.1 of Ref. 3], there is a slight tension between the lensing amplitudes that are inferred from the trispectrum and from the power spectrum. In particular, while the former is consistent with the value expected in $\Lambda \mathrm{CDM}$, the temperature power spectrum shows a mild preference for a higher lensing power. Since the effect of increasing the neutrino mass is similar to that of a smaller lensing amplitude (as both result in a suppression of power at small scales), including the lensing likelihood tends to shift the value of the total

\footnotetext{
${ }^{3}$ See for instance the fitting functions provided in Ref. [68, extracted from the numerical results of the PArthENopE BBN code 69.
}

neutrino mass to larger values [3]. Instead, the inclusion of the lensing likelihood does not change significantly the constraints on the effective number of relativistic degrees of freedom, at least for the $\Lambda \mathrm{CDM}$ model.

Another piece of information that is in tension with the corresponding Planck estimate is the value of the Hubble constant inferred from astrophysical measurements, as discussed in Sec. 5.3 of Ref. 3]. This includes the HST value used in our analysis, $H_{0}=73.8 \pm 2.4$ $\mathrm{km} / \mathrm{s} / \mathrm{Mpc}$, that is discrepant with the Planck $\Lambda \mathrm{CDM}$ estimate $H_{0}=67.3 \pm 1.2 \mathrm{~km} / \mathrm{s} / \mathrm{Mpc}$ at more than $2 \sigma$, although it should always be remembered that CMB estimates are highly model dependent. The reasons for this discrepancy are, to date, not yet well understood and are a matter of intense debate in the community. It is however possible that this tension is relieved in some extensions of the standard $\Lambda \mathrm{CDM}$ model. For this reason, we have decided to consider the HST data in some of our enlarged datasets.

Finally, we use the $\sigma_{8}$ measurements from the CHFTLens survey and from the Planck SZ cluster counts, as reported in Sec. II A4. These values are however both discrepant with the value estimated from Planck CMB at the $2 \sigma$ level (see discussion in Sec. 5.5 of Ref. [3]). This tension has not yet been explained either, but it could be related to the difficulties in adequately modelling selection biases and calibrating cluster masses. As in the case of the Hubble constant, however, there is the possibility that the discrepancy is alleviated in some extended cosmological models (like for example those that include the neutrino mass as a free parameter). Following the same rationale as for the inclusion of the HST data, we have derived constraints from enlarged datasets that include the $\sigma_{8}$ measurements. These should however be regarded as quite un-conservative.

\section{RESULTS}

\section{A. Massive neutrinos}

We present here the results on our baseline scenario with three active neutrino degenerate species. Table II depicts the $95 \%$ CL constraints on the sum of the three active neutrino masses $\sum m_{\nu}$. Notice that, without the inclusion of the constraints on $\sigma_{8}$ and $\Omega_{m}$ the upper limits on the neutrino mass are mostly driven by the new BOSS DR11 BAO measurements, being the tightest limit $\sum m_{\nu}<0.22 \mathrm{eV}$ at $95 \% \mathrm{CL}$ from the combination of CMB data, BAO and HST measurements of the Hubble constant. However, since there exists a well known discrepancy on the measured value of $H_{0}$ from the Planck and the HST experiments 3 , we have also considered the combination of $\mathrm{CMB}$ and $\mathrm{BAO}$ data with SNLS Supernovae Ia luminosity distance measurements. Such a combination provides an upper 95\% CL limit of $\sum m_{\nu}<0.23 \mathrm{eV}$, in perfect agreement with the findings of the recent BOSS results [56 using the full shape of 


\begin{tabular}{|c|c|c|c|c|c|c|c|c|}
\hline & CMB+DR11 & $\begin{array}{c}\text { CMB+DR11 } \\
+ \text { HST } \\
\end{array}$ & $\begin{array}{c}\text { CMB+DR11 } \\
+\mathrm{WZ} \\
\end{array}$ & $\begin{array}{l}\text { CMB+DR11 } \\
+\mathrm{WZ}+\mathrm{HST}\end{array}$ & $\begin{array}{c}\text { CMB+ DR11 } \\
+\mathrm{WZ}+\mathrm{BAO}+\mathrm{HST} \\
\end{array}$ & $\begin{array}{c}\text { CMB+DR11 } \\
+ \text { BAO } \\
\end{array}$ & $\begin{array}{l}\text { CMB+DR11 } \\
+ \text { BAO+HST }\end{array}$ & $\begin{array}{l}\text { CMB+ DR11 } \\
+\mathrm{BAO}+\text { SNLS } \\
\end{array}$ \\
\hline$\Sigma m_{\nu}[\mathrm{eV}]$ & $<0.25$ & $<0.22$ & $<0.25$ & $<0.23$ & $<0.24$ & $<0.26$ & $<0.22$ & $<0.23$ \\
\hline \multicolumn{9}{|c|}{$\begin{array}{l}\text { SZ Clusters \& } \\
\text { CFHTLens }\end{array}$} \\
\hline$\Sigma m_{\nu}[\mathrm{eV}]$ & $0.30_{-0.14}^{+0.12}$ & $0.25_{-0.13}^{+0.12}$ & $0.27_{-0.13}^{+0.14}$ & $0.25_{-0.11}^{+0.10}$ & $0.26_{-0.13}^{+0.18}$ & $0.29_{-0.12}^{+0.13}$ & $0.24_{-0.12}^{+0.10}$ & $0.27_{-0.13}^{+0.12}$ \\
\hline \multicolumn{9}{|c|}{ SZ Clusters } \\
\hline$\Sigma m_{\nu}[\mathrm{eV}]$ & $0.30_{-0.14}^{+0.12}$ & $0.25_{-0.13}^{+0.13}$ & $0.27_{-0.13}^{+0.12}$ & $0.24_{-0.10}^{+0.10}$ & $0.25_{-0.13}^{+0.17}$ & $0.29_{-0.12}^{+0.13}$ & $0.23_{-0.12}^{+0.10}$ & $0.27_{-0.13}^{+0.11}$ \\
\hline \multicolumn{9}{|l|}{ CFHTLens } \\
\hline$\Sigma m_{\nu}[\mathrm{eV}]$ & $<0.33$ & $<0.28$ & $<0.30$ & $<0.27$ & $<0.28$ & $<0.33$ & $<0.27$ & $<0.30$ \\
\hline
\end{tabular}

TABLE II: $95 \%$ CL constraints on the sum of the neutrino masses, $\Sigma m_{\nu}$, from the different combinations of data sets explored here.

the clustering correlation function. The addition of the constraints on $\sigma_{8}$ and $\Omega_{m}$ from the CFHTLens survey displaces the bounds on the neutrino mass to higher values, the reason for that being the lower $\sigma_{8}$ preferred by CFHTLens weak lensing measurements. Due the poor constraining power of the weak lensing data the neutrino mass bounds are not significantly altered. On the other hand, when adding the constraint on $\sigma_{8}$ and $\Omega_{m}$ from the Planck-SZ cluster catalog on galaxy number counts, a non zero value for the sum of the three active neutrino masses of $\sim 0.3 \mathrm{eV}$ is favoured at $4 \sigma$. In particular, the combination of $\mathrm{CMB}$ data with $\mathrm{BAO}$ measurements from BOSS DR11, WiggleZ power spectrum (full shape) data and a prior on $H_{0}$ from HST after considering the inclusion of Planck SZ clusters information leads to the value $\sum m_{\nu}=0.24_{-0.10}^{+0.10} \mathrm{eV}$ at $95 \% \mathrm{CL}$. The combination of weak lensing data and galaxy number counts data is mostly driven by the latter and therefore the constraints do not change significantly with respect to the case in which the analyses are performed with galaxy cluster counts information only. A similar effect, although in a slightly different scenario and different data sets, was found by Refs. [48, 49.

Figure 1 illustrates our findings for three possible data combinations: CMB data, combined with BOSS DR11 $\mathrm{BAO}$ measurements, additional $\mathrm{BAO}$ measurements and a prior on the Hubble constant from HST (depicted by the blue contours); and the same data combination but considering also the $\sigma_{8}-\Omega_{m}$ weak lensing (galaxy number counts) constraint (depicted by the red (green) contours). The left panel depicts the very well known degeneracy in the $\left(\sum m_{\nu}(\mathrm{eV}), H_{0}\right)$ plane, showing the $68 \%$ and $95 \%$ CL allowed contours by the different data sets specified above. Considering CMB data only, a higher value of $\sum m_{\nu}$ can be compensated by a decrease on the
Hubble constant $H_{0}$ since the shift induced in the distance to the last scattering surface caused by a larger $\sum m_{\nu}$ can be compensated by a lower $H_{0}$. Notice that when Planck SZ cluster information on the $\sigma_{8}-\Omega_{m}$ relationship is added, the allowed neutrino mass regions are displaced and a non zero value for the sum of the three active neutrino masses is favoured at $\sim 4 \sigma$. The right panel of Fig. 1 1 shows the $68 \%$ and $95 \%$ CL allowed regions in the $\left(\sum m_{\nu}(\mathrm{eV}), \sigma_{8}\right)$ plane. The allowed contours of both $\sigma_{8}$ and $\sum m_{\nu}$ are considerably displaced after considering Planck clusters data. The power spectrum normalization $\sigma_{8}$ has smaller values when neutrinos are massive (due to the neutrino free streaming nature), being precisely these smaller values of $\sigma_{8}$ those preferred by galaxy cluster number counts.

\section{B. Massive neutrinos and thermal axions}

In this section we present the constraints on a scenario including both massive neutrinos and a thermal axion. Table III presents the constraints on the sum of the three active neutrino masses and on the axion mass (both in eV) for the different cosmological data combinations considered here. Notice that BBN bounds are also quoted here since a thermal axion will also contribute to the extra radiation component at the BBN period, by an amount given by:

$$
\Delta N_{\mathrm{eff}}=\frac{4}{7}\left(\frac{3}{2} \frac{n_{a}}{n_{\nu}}\right)^{4 / 3}
$$

being $n_{a}$ the current axion number density and $n_{\nu}=$ $112 \mathrm{~cm}^{-3}$, the current number density of each active neutrino plus antineutrino flavour. We have applied the BBN 

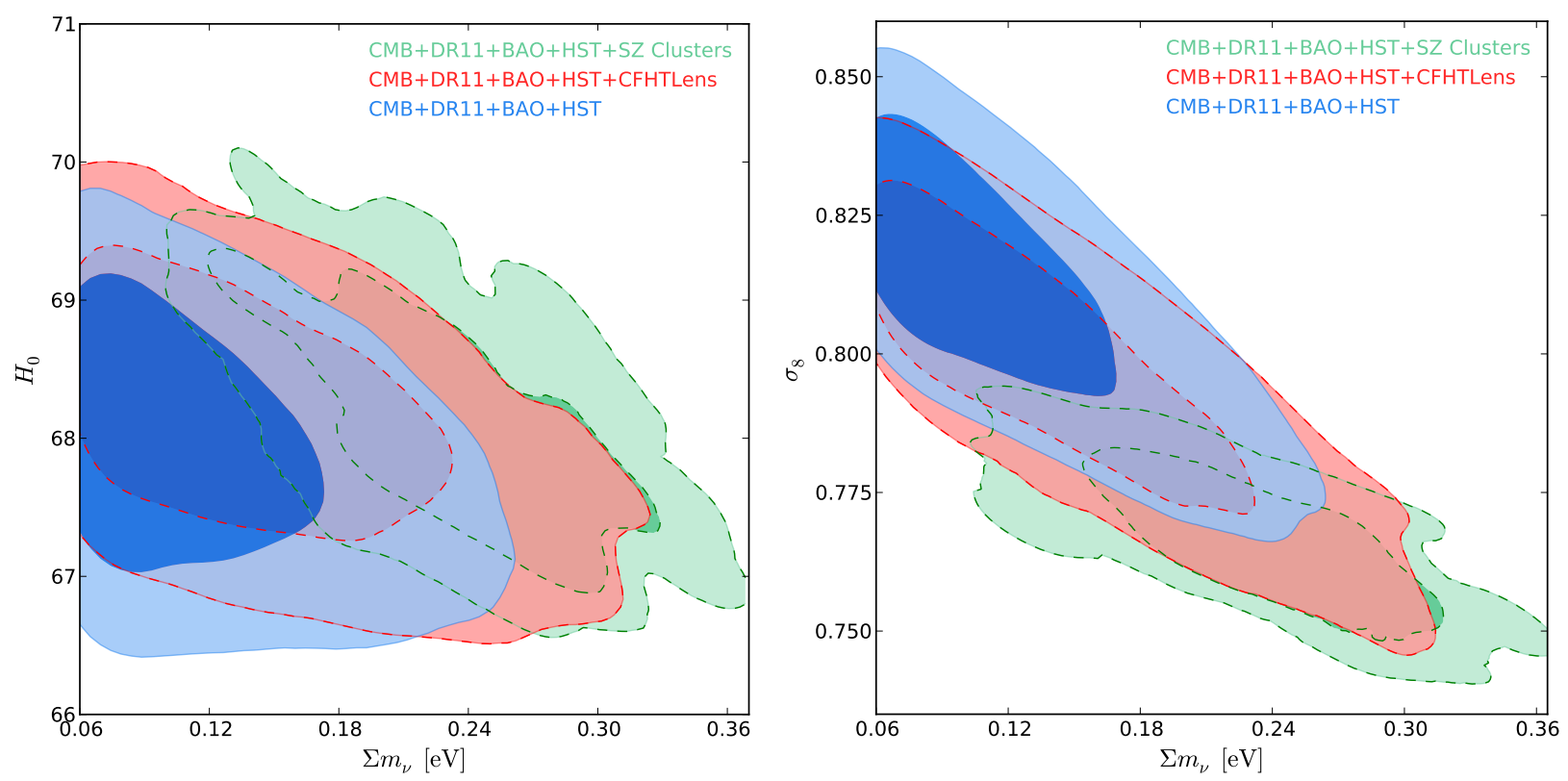

FIG. 1: Left panel: the blue contours show the $68 \%$ and $95 \%$ CL allowed regions from the combination of CMB data, BOSS DR11 BAO measurements, additional BAO measurements and a prior on the Hubble constant from $\mathrm{HST}$ in the $\left(\sum m_{\nu}(\mathrm{eV})\right.$, $\left.H_{0}\right)$ plane. The red (green) contours depict the results when the $\sigma_{8}-\Omega_{m}$ weak lensing (galaxy number counts) constraint is added in the analysis. Right panel: as in the left panel but in the $\left(\sum m_{\nu}(\mathrm{eV}), \sigma_{8}\right)$ plane.

consistency relation in our MCMC analyses of Planck data, to compute the Helium mass fraction as a function of $\Delta N_{\text {eff. }}$. Nevertheless the bounds on neutrino and axion masses are not significantly affected if the Helium mass fraction is kept fixed for CMB purposes. Notice that, before applying constraints from Planck SZ Clusters or CHFTLens constraints on the $\sigma_{8}-\Omega_{m}$ relationship, the most stringent $95 \%$ CL bounds, without including BBN bounds, are $\sum m_{\nu}<0.21 \mathrm{eV}$ and $m_{a}<0.59 \mathrm{eV}$, considering CMB, BOSS BAO DR11, additional BAO measurements, WiggleZ power spectrum (full shape) information and the $H_{0}$ HST prior. These bounds are in perfect agreement with the findings of Ref. 36, albeit they are slightly tighter, mostly due to the more accurate new BOSS BAO measurements.

After considering BBN bounds with deuterium estimates from 52 (68]) and helium constraints from Ref. [53, which constrain the contribution of the thermal axion to the relativistic degrees of freedom at the BBN epoch, the $95 \%$ CL bounds quoted above traslate into $\sum m_{\nu}<0.25 \mathrm{eV}$ and $m_{a}<0.57 \mathrm{eV}\left(\sum m_{\nu}<0.21 \mathrm{eV}\right.$ and $\left.m_{a}<0.61 \mathrm{eV}\right)$.

The addition of weak lensing constraints on the $\sigma_{8}-\Omega_{m}$ relationship from the CFHTLens experiment makes the neutrino and axion mass bounds weaker, due to the lower $\sigma_{8}$ preferred by the former data set, which favours higher values for the thermal relic masses. If further information on the $\sigma_{8}-\Omega_{m}$ relationship from the Planck SZ cluster number counts is considered in the MCMC analyses, there exists evidence for a neutrino mass of $\sim 0.2 \mathrm{eV}$ at the $\sim 3 \sigma$ level exclusively for the case in which CMB data is combined with BOSS BAO DR11 measurements and full-shape power spectrum information from the WiggleZ galaxy survey. There exists as well a mild evidence $(\sim 2 \sigma)$ for an axion mass of $0.6 \mathrm{eV}$ for two isolated cases in which either the HST $H_{0}$ prior or SNIa luminosity distance measurements are considered in combination with all the BAO measurements here exploited. However, there is no evidence for neutrino and axions masses simultaneously.

Figure 2, left panel, depicts the $68 \%$ and $95 \%$ CL allowed regions arising from the combination of CMB data, BOSS DR11 BAO measurements, additional BAO measurements and a prior on the Hubble constant from HST in the $\left(\sum m_{\nu}(\mathrm{eV}), m_{a}(\mathrm{eV})\right)$ plane. Once the Planck SZ cluster number counts information on the $\sigma_{8}-\Omega_{m}$ relationship is added, a non zero value of the axion mass is favoured by data at the $\sim 2.2 \sigma$. The right panel of Fig. 2 shows the $68 \%$ and $95 \%$ CL contours in the $\left(\sum m_{\nu}(\mathrm{eV})\right.$, $m_{a}(\mathrm{eV})$ ) plane resulting from the analysis of CMB data, BOSS DR11 BAO measurements, additional BAO measurements - except for the WiggleZ galaxy survey information which is removed and considered in its full-shape form - and the HST $H_{0}$ prior. Notice that no evidence for non-zero neutrino masses nor for non-zero axion mass appears in this case. 


\begin{tabular}{|c|c|c|c|c|c|c|c|c|}
\hline & CMB+DR11 & $\begin{array}{c}\text { CMB+DR11 } \\
+ \text { HST }\end{array}$ & $\begin{array}{c}\text { CMB+DR11 } \\
+\mathrm{WZ}\end{array}$ & $\begin{array}{l}\text { CMB+DR11 } \\
+\mathrm{WZ}+\mathrm{HST}\end{array}$ & $\begin{array}{c}\text { CMB+DR11 } \\
+\mathrm{WZ}+\mathrm{BAO}+\mathrm{HST}\end{array}$ & $\begin{array}{c}\text { CMB+DR11 } \\
+ \text { BAO }\end{array}$ & $\begin{array}{l}\text { CMB+DR11 } \\
+\mathrm{BAO}+\mathrm{HST}\end{array}$ & $\begin{array}{c}\text { CMB+DR11 } \\
+ \text { BAO+SNLS }\end{array}$ \\
\hline$\Sigma m_{\nu}[\mathrm{eV}]$ & $<0.24$ & $<0.21$ & $<0.24$ & $<0.22$ & $<0.21$ & $<0.23$ & $<0.20$ & $<0.22$ \\
\hline$m_{a}[\mathrm{eV}]$ & $<0.79$ & $<0.77$ & $<0.65$ & $<0.62$ & $<0.59$ & $<0.74$ & $<0.75$ & $<0.76$ \\
\hline
\end{tabular}

SZ Clusters \&

CFHTLensing

\begin{tabular}{|c|c|c|c|c|c|c|c|c|}
\hline$\Sigma m_{\nu}[\mathrm{eV}]$ & $<0.36$ & $<0.27$ & $0.21_{-0.13}^{+013}$ & $<0.32$ & $<0.30$ & $<0.31$ & $<0.28$ & $<0.31$ \\
\hline$m_{a}[\mathrm{eV}]$ & $<1.08$ & $<1.09$ & $<0.88$ & $<0.81$ & $<0.77$ & $<1.12$ & $0.63_{-0.49}^{+0.47}$ & $0.58_{-0.48}^{+0.50}$ \\
\hline \multicolumn{9}{|l|}{ SZ Clusters } \\
\hline$\Sigma m_{\nu}[\mathrm{eV}]$ & $<0.36$ & $<0.27$ & $0.20_{-0.14}^{+013}$ & $<0.32$ & $<0.30$ & $<0.31$ & $<0.27$ & $<0.31$ \\
\hline$m_{a}[\mathrm{eV}]$ & $<1.07$ & $<1.07$ & $<0.87$ & $<0.81$ & $<0.77$ & $<1.10$ & $0.62_{-0.48}^{+0.46}$ & $0.57_{-0.47}^{+0.50}$ \\
\hline \multicolumn{9}{|l|}{ CFHTLens } \\
\hline$\Sigma m_{\nu}[\mathrm{eV}]$ & $<0.29$ & $<0.24$ & $<0.28$ & $<0.25$ & $<0.25$ & $<0.27$ & $<0.24$ & $<0.26$ \\
\hline$m_{a}[\mathrm{eV}]$ & $<0.94$ & $<0.95$ & $<0.74$ & $<0.68$ & $<0.67$ & $<0.96$ & $<0.94$ & $<0.98$ \\
\hline \multicolumn{9}{|l|}{$\mathrm{BBN}$} \\
\hline$\Sigma m_{\nu}[\mathrm{eV}](D / H)_{p} 52$ & $<0.27$ & $<0.24$ & $<0.26$ & $<0.27$ & $<0.25$ & $<0.27$ & $<0.23$ & $<0.24$ \\
\hline$\Sigma m_{\nu}[\mathrm{eV}](D / H)_{p} 68$ & $<0.24$ & $<0.20$ & $<0.23$ & $<0.21$ & $<0.21$ & $<0.22$ & $<0.20$ & $<0.22$ \\
\hline$m_{a}[\mathrm{eV}](D / H)_{p}[52]$ & $<1.15$ & $<0.76$ & $<0.60$ & $<0.59$ & $<0.57$ & $<0.79$ & $<0.77$ & $<1.38$ \\
\hline$m_{a}[\mathrm{eV}](D / H)_{p} \underline{68}$ & $<0.82$ & $<0.80$ & $<0.67$ & $<0.64$ & $<0.61$ & $<0.77$ & $<0.78$ & $<0.79$ \\
\hline
\end{tabular}

TABLE III: 95\% CL constraints on the sum of the neutrino masses, $\Sigma m_{\nu}$, and on the axion mass, $m_{a}$, both in eV, from the different combinations of data sets explored here. When BBN bounds are included, the first (second) raw refers to the constraints obtained combining the primordial deuterium values from Ref. [52] $\left([68)(D / H)_{p}=(2.53 \pm 0.04) \times 10^{-5}\right.$ $\left((D / H)_{p}=(2.87 \pm 0.22) \times 10^{-5}\right)$ with measurements of the helium mass fraction $Y_{p}=0.254 \pm 0.003$ from Ref. [53].

\section{Massive neutrinos and extra dark radiation species}

We first report here the constraints resulting when considering both massive neutrinos and $\Delta N_{\text {eff }}$ massless dark radiation species. These massless species may appear in extensions of the Standard Model of elementary particles containing a dark sector, as, for instance, in the so-called asymmetric dark matter scenarios. In all these models, when the value of $N_{\text {eff }}$ is larger than the canonical 3.046, $\Delta N_{\text {eff }}=N_{\text {eff }}-3.046$ is related to the extra density in massless hot relics. On the other hand, if the value of $N_{\text {eff }}$ is smaller than the standard 3.046, the active neutrino temperature is reduced and there are no extra massless species.

Table IV depicts the $95 \%$ CL constraints on the sum of the three active neutrino masses $\sum m_{\nu}$ and well as on the total number of dark radiation species $N_{\text {eff }}$, corresponding to the contribution from the three active neutrinos plus $\Delta N_{\text {eff }}$ massless dark radiation species, for the different data combinations explored here. The bounds on the neutrino mass are less stringent than in standard three neutrino massive case due to the large degeneracy between $\sum m_{\nu}$ and $N_{\text {eff }}$, since a larger number of massless sterile neutrino-like species will increase the radiation content of the universe, and, in order to leave unchanged both the matter-radiation equality era and the location of the CMB acoustic peaks, the matter content of the universe must also increase, allowing therefore for larger neutrino masses. We find $\sum m_{\nu}<0.31 \mathrm{eV}$ and $N_{\text {eff }}=3.45_{-0.54}^{+0.59}$ at $95 \%$ CL from the combination of CMB data and BOSS DR11 BAO measurements. 

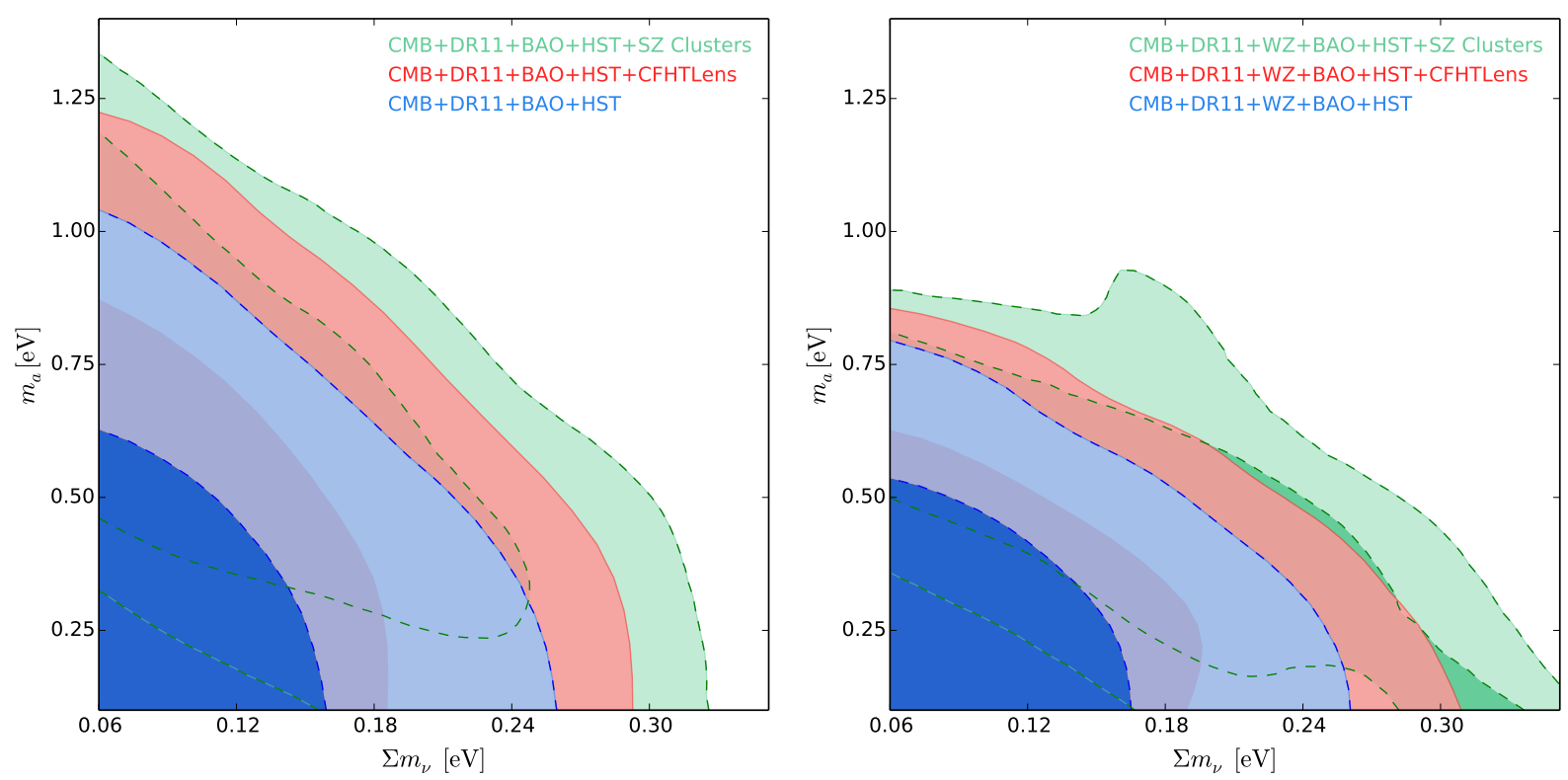

FIG. 2: Left panel: the blue contours show the $68 \%$ and $95 \%$ CL allowed regions from the combination of CMB data, BOSS DR11 BAO measurements, additional BAO measurements and a prior on the Hubble constant from HST (depicted by the blue contours) in the $\left(\sum m_{\nu}(\mathrm{eV}), m_{a}(\mathrm{eV})\right)$ plane. The red (green) contours depict the results when the $\sigma_{8}-\Omega_{m}$ weak lensing (galaxy number counts) constraint is added in the analysis. Right panel: as in the left panel but replacing the WiggleZ BAO geometrical information by the WiggleZ full-shape matter power spectrum measurements.

When the prior on the value of the Hubble constant from HST is included in the analyses, the mean value of $N_{\text {eff }}$ and the bound on the neutrino masses are both mildly larger accordingly to the larger value of $H_{0}$ preferred by HST data. The Hubble constant $H_{0}$ and $N_{\text {eff }}$ are positively correlated through measurements of the CMB, see Ref. [71 for a complete description of the effects of $N_{\text {eff }}$ on the CMB . If the value of $N_{\text {eff }}$ is increased, in order to keep fixed both the angular location of the acoustic peaks and the matter-radiation equality epoch (to leave unchanged the first peak height via the early ISW effect), the expansion rate is also increased, implying therefore a larger $H_{0}$ and a shorter age of the Universe at recombination.

Since HST measurements point to a higher $H_{0}$ value, a larger value of $N_{\text {eff }}$ will be favoured by data, which also implies a higher neutrino mass bound due to the strong $\sum m_{\nu}-N_{\text {eff }}$ degeneracy. The $95 \%$ CL constraints from the combination of CMB data, BOSS DR11 BAO measurements and the HST $H_{0}$ prior are $\sum m_{\nu}<0.34 \mathrm{eV}$ and $N_{\text {eff }}=3.57_{-0.48}^{+0.45}$. Once the Hubble constant prior from the HST experiment is added in the analyses, there exists a very mild preference $(2 \sigma)$ for a value of $N_{\text {eff }}$ larger than the canonical expectation of 3.046, agreeing as well with the results of Ref. 3 .

The addition of the measurements of the deuterium (either from older estimates 68, or from the most recent measurements from Ref. [52]) and the helium [53] light element abundances, reduce both the mean value and the errors of $N_{\text {eff }}$ significantly. After the addition of BBN bounds the errors on $N_{\text {eff }}$ are reduced by a half. Ta- ble IV contains the BBN constraints obtained using the fitting functions for the theoretical deuterium and helium primordial abundances, as a function of $\Omega_{b} h^{2}$ and $N_{\text {eff }}$, of Ref. 68 (extracted from the numerical results of the PArthENopE BBN code [69). We report in the table exclusively these constraints because they are the most conservative ones: we find $\sum m_{\nu}<0.24 \mathrm{eV}$ and $N_{\text {eff }}=3.25_{-0.24}^{+0.25}$ at $95 \%$ CL from the analysis of CMB data, WiggleZ power spectrum measurements, the HST $H_{0}$ prior and BBN light elements abundances information (with the deuterium measurements from Ref. [52]). Notice that there is no evidence for $N_{\text {eff }}>3$ when considering the most recent estimates of primordial deuterium abundances. However, if we consider instead previous measurements of deuterium, as those from Ref. [68, there exists a $3.5-4 \sigma$ preference for $N_{\text {eff }}>3$ if HST data is included in the analyses. Without the inclusion of HST data the preference for $N_{\text {eff }}>3$ still persists, albeit at the $2.5-3 \sigma$ CL. As previously stated, the BBN bounds on $N_{\text {eff }}$ and $\sum m_{\nu}$ quoted in Tab. IV are the most conservative ones we found. Different bounds are obtained if an alternative fitting function is used in order to compute the theoretical deuterium and helium primordial abundances. We have performed as well such an exercise, using the fitting functions from Refs. [52, 72 and, in general, the mean value obtained for $N_{\text {eff }}$ is larger than the constraints quoted above. In the case in which recent deuterium measurements are considered in the analysis, the mean value of $N_{\text {eff }}$ is displaced by $\sim 2 \sigma$ with respect to the mean values obtained when using the fitting function of 68. If previous deuterium mea- 


\begin{tabular}{|c|c|c|c|c|c|c|c|c|}
\hline & CMB+DR11 & $\begin{array}{c}\text { CMB+DR11 } \\
+ \text { HST } \\
\end{array}$ & $\begin{array}{c}\text { CMB+DR11 } \\
\text { +WZ }\end{array}$ & $\begin{array}{l}\text { CMB+DR11 } \\
+ \text { WZ+HST }\end{array}$ & $\begin{array}{c}\text { CMB+DR11 } \\
+\mathrm{WZ}+\mathrm{BAO}+\mathrm{HST} \\
\end{array}$ & $\begin{array}{c}\text { CMB+DR11 } \\
+ \text { BAO }\end{array}$ & $\begin{array}{l}\text { CMB+DR11 } \\
+ \text { BAO+HST }\end{array}$ & $\begin{array}{c}\text { CMB+DR11 } \\
+ \text { BAO+SNLS } \\
\end{array}$ \\
\hline$\Sigma m_{\nu}[\mathrm{eV}]$ & $<0.31$ & $<0.31$ & $<0.32$ & $<0.34$ & $<0.34$ & $<0.31$ & $<0.31$ & $<0.29$ \\
\hline$N_{\text {eff }}$ & $3.45_{-0.54}^{+0.59}$ & $3.66_{-0.49}^{+0.52}$ & $3.32_{-0.62}^{+0.55}$ & $3.57_{-0.48}^{+0.50}$ & $3.56_{-0.49}^{+0.45}$ & $3.43_{-0.59}^{+0.58}$ & $3.66_{-0.47}^{+0.48}$ & $3.48_{-0.56}^{+0.58}$ \\
\hline
\end{tabular}

SZ Clusters \&

CFHTLensing

\begin{tabular}{|c|c|c|c|c|c|c|c|c|}
\hline$\Sigma m_{\nu}[\mathrm{eV}] \&$ & $0.37_{-0.18}^{+0.24}$ & $0.37_{-0.20}^{+0.20}$ & $0.32_{-0.19}^{+0.19}$ & $0.35_{-0.17}^{+0.16}$ & $0.37_{-0.17}^{+0.26}$ & $0.32_{-0.21}^{+0.18}$ & $0.37_{-0.20}^{+0.18}$ & $0.32_{-0.17}^{+0.15}$ \\
\hline$N_{\text {eff }}$ & $3.32_{-0.55}^{+0.53}$ & $3.54_{-0.54}^{+0.48}$ & $3.24_{-0.70}^{+0.58}$ & $3.56_{-0.59}^{+0.59}$ & $3.56_{-0.60}^{+1.09}$ & $3.17_{-0.59}^{+0.64}$ & $3.54_{-0.62}^{+60}$ & $3.25_{-0.43}^{+0.47}$ \\
\hline
\end{tabular}

SZ Clusters

\begin{tabular}{lrrrrrrrr}
\hline$\Sigma m_{\nu}[\mathrm{eV}]$ & $0.37_{-0.19}^{+0.24}$ & $0.36_{-0.18}^{+0.18}$ & $0.32_{-0.19}^{+0.19}$ & $0.35_{-0.16}^{+0.17}$ & $0.36_{-0.18}^{+0.26}$ & $0.32_{-0.20}^{+0.18}$ & $0.37_{-0.21}^{+0.18}$ & $0.32_{-0.16}^{+0.15}$ \\
$N_{\text {eff }}$ & $3.33_{-0.53}^{+0.55}$ & $3.55_{-0.58}^{+0.51}$ & $3.25_{-0.68}^{+0.57}$ & $3.56_{-0.58}^{+0.59}$ & $3.55_{-0.59}^{+0.65}$ & $3.18_{-0.59}^{+0.63}$ & $3.54_{-0.59}^{+0.62}$ & $3.25_{-0.44}^{+0.49}$ \\
\hline
\end{tabular}

\section{CFHTLens}

\begin{tabular}{|c|c|c|c|c|c|c|c|c|}
\hline$\Sigma m_{\nu}[\mathrm{eV}]$ & $<0.41$ & $<0.44$ & $<0.39$ & $<0.41$ & $<0.42$ & $<0.40$ & $<0.43$ & $<0.39$ \\
\hline$N_{\text {eff }}$ & $3.39_{-0.55}^{+0.57}$ & $3.59_{-0.54}^{+0.52}$ & $3.28_{-0.63}^{+0.58}$ & $3.55_{-0.47}^{+0.53}$ & $3.54_{-0.47}^{+0.52}$ & $3.33_{-0.61}^{+0.61}$ & $3.58_{-0.50}^{+0.50}$ & $3.37_{-0.55}^{+0.58}$ \\
\hline \multicolumn{9}{|l|}{$\mathrm{BBN}$} \\
\hline$\Sigma m_{\nu}[\mathrm{eV}](D / H)_{p} 52$ & $<0.27$ & $<0.29$ & $<0.29$ & $<0.24$ & $<0.25$ & $<0.28$ & $<0.32$ & $<0.32$ \\
\hline$\Sigma m_{\nu}[\mathrm{eV}](D / H)_{p} 68$ & $<0.30$ & $<0.28$ & $<0.32$ & $<0.31$ & $<0.32$ & $<0.31$ & $<0.28$ & $<0.28$ \\
\hline$N_{\text {eff }}(D / H)_{p} 52$ & $3.17_{-0.27}^{+0.26}$ & $3.24_{-0.25}^{+0.26}$ & $3.13_{-0.29}^{+0.30}$ & $3.25_{-0.24}^{+0.25}$ & $3.22_{-0.25}^{+0.27}$ & $3.11_{-0.31}^{+0.31}$ & $3.23_{-0.26}^{+0.27}$ & $3.18_{-0.31}^{+0.29}$ \\
\hline$N_{\text {eff }}(D / H)_{p} 68$ & $3.47_{-0.34}^{+0.35}$ & $3.56_{-0.33}^{+0.34}$ & $3.52_{-0.31}^{+0.33}$ & $3.52_{-0.26}^{+0.27}$ & $3.52_{-0.32}^{+0.33}$ & $3.48_{-0.36}^{+0.35}$ & $3.57_{-0.33}^{+0.34}$ & $3.49_{-0.35}^{+0.36}$ \\
\hline
\end{tabular}

TABLE IV: 95\% CL constraints on the sum of the neutrino masses, $\Sigma m_{\nu}$, in eV, and on the relativistic degrees of freedom $N_{\text {eff }}$ from the different combinations of data sets explored here. When BBN bounds are included, the first (second) raw refers to the constraints obtained combining the primordial deuterium values from Ref. [52 $(68])(D / H)_{p}=(2.53 \pm 0.04) \times 10^{-5}$ $\left((D / H)_{p}=(2.87 \pm 0.22) \times 10^{-5}\right)$ with measurements of the helium mass fraction $Y_{p}=0.254 \pm 0.003$ from Ref. [53]

surements 68. are used for our numerical analyses, the mean value of $N_{\text {eff }}$ is also mildly larger than the mean $N_{\text {eff }}$ values obtained when applying the fitting functions from Ref. 68. The upper bound on the sum of the three active neutrino masses is also larger for the two analyses (with recent and previous deuterium measurements), due to the degeneracy between $N_{\text {eff }}$ and $\sum m_{\nu}$. As an example, from the analysis of CMB data, WiggleZ power spectrum measurements, the HST $H_{0}$ prior and BBN light elements abundances information (with recent deuterium measurements from Ref. [52]), our analysis point to the following values: $N_{\text {eff }}=3.47_{-0.27}^{+0.27}$ and $\sum m_{\nu}<0.30 \mathrm{eV}$, both at $95 \% \mathrm{CL}$. If previous measurements of deuterium are instead considered [68, the $95 \%$ CL limits are $N_{\text {eff }}=3.60_{-0.32}^{+0.33}$ and $\sum m_{\nu}<0.32 \mathrm{eV}$.
Therefore, a preference for $N_{\text {eff }}>3$ at the $3.5-4 \sigma$ $(2.5-3 \sigma)$ CL with (without) the HST $H_{0}$ prior included in the analyses will always be present in the results obtained with the fitting functions of Refs. [52, 772, independently of the deuterium measurements exploited.

As in the standard three massive neutrino case, the addition of the constraints on the $\sigma_{8}$ and $\Omega_{m}$ cosmological parameters from the CFHTLens survey displaces the bounds on the neutrino mass to higher values. When adding the $\sigma_{8}-\Omega_{m}$ relationship from the Planck-SZ cluster catalog on galaxy number counts, a non zero value for the sum of the three active neutrino masses of $\sim 0.35 \mathrm{eV}$ is favoured at $4 \sigma$. Notice that in this case the preferred mean value for $\sum m_{\nu}$ is higher than in the three massive neutrino case due to the fact that $N_{\text {eff }}$ is a free pa- 
rameter and there exists a large degeneracy among $N_{\text {eff }}$ and $\sum m_{\nu}$. The combination of CMB data with BAO measurements from BOSS DR11, WiggleZ power spectrum (full shape) data and a prior on $H_{0}$ from HST after considering the inclusion of Planck SZ clusters information leads to the values $\sum m_{\nu}=0.35_{-0.16}^{+0.17} \mathrm{eV}$ and $N_{\text {eff }}=3.56_{-0.58}^{+0.59}$ at $95 \% \mathrm{CL}$.

The bounds quoted above have been obtained using the $\mathrm{BBN}$ theoretical prediction for helium in the CMB data analysis. However, it is also possible to fix the helium fraction $Y_{p}$ in the Monte Carlo Markov Chain analyses of $\mathrm{CMB}$ data and assume that $Y_{p}$ is an independent parameter constrained by $\mathrm{BBN}$ observations only. We have also performed such an exercise, fixing $Y_{p}=0.24$, and we find, in general, larger values for both the mean value of $N_{\text {eff }}$ and its errors, and, consequently, a slightly larger bound on the neutrino mass, due to the $\sum m_{\nu}-N_{\text {eff }}$ degeneracy. In particular, we find $\sum m_{\nu}<0.32 \mathrm{eV}$ and $N_{\text {eff }}=3.60_{-0.65}^{+0.67}$ at $95 \%$ CL from the combination of CMB data and BOSS DR11 BAO measurements, and $\sum m_{\nu}<0.34 \mathrm{eV}$ and $N_{\text {eff }}=3.84_{-0.56}^{+0.60}$ at $95 \% \mathrm{CL}$ if a prior from HST on the Hubble constant $H_{0}$ is added to the former data combination. These findings agree with the results of Ref. [15, where it is also found that the BBN consistency relation leads to a constraint on $N_{\text {eff }}$ closer to the canonical value of 3.046 than in the case of fixing $Y_{p}=0.24$. Once $\mathrm{BBN}$ measurements are considered in the data analyses, the differences between the analyses with and without the BBN consistency relation included become irrelevant.

Figure 3, left panel, shows the degeneracy between the $\sum m_{\nu}$ and the total number of dark radiation species $N_{\text {eff }}$ (which accounts for the contribution of the three active neutrino species plus $\Delta N_{\text {eff }}$ massless sterile neutrino-like species). The red contours depict the $68 \%$ and $95 \% \mathrm{CL}$ allowed regions resulting from the combination of $\mathrm{CMB}$, BOSS DR11 BAO measurements, and previous BAO measurements. As the value of $N_{\text {eff }}$ increases, a larger neutrino mass is allowed, to leave unchanged both the matter radiation equality era and the angular location of the acoustic peaks, as well as the high of the first acoustic peak via the early ISW effect. The blue region denotes the results considering the HST $H_{0}$ prior as well in the analysis: notice that the allowed regions are shifted towards higher values of $N_{\text {eff }}$. Figure 3 , right panel, illustrates the degeneracy between $N_{\text {eff }}$ and the Hubble constant $H_{0}$. The color coding is identical to the one used in the figure shown in the left panel, in which the red contours are related to the $68 \%$ and $95 \%$ CL allowed regions from the combination of CMB data, BOSS DR11 $\mathrm{BAO}$ measurements and additional BAO measurements and the blue regions refer to the constraints after adding a prior on the Hubble constant from the HST experiment.

\section{Massive neutrinos and extra massive sterile neutrino species}

The latest possibility for thermal relics explored in this study is the case in which there exists three active light massive neutrinos plus one massive sterile neutrino species characterised by an effective mass $m_{s}^{\text {eff }}$, which reads

$$
m_{s}^{\mathrm{eff}}=\left(T_{s} / T_{\nu}\right)^{3} m_{s}=\left(\Delta N_{\mathrm{eff}}\right)^{3 / 4} m_{s},
$$

being $T_{s}\left(T_{\nu}\right)$ the current temperature of the sterile (active) neutrino species, $\Delta N_{\text {eff }} \equiv N_{\text {eff }}-3.046=\left(T_{s} / T_{\nu}\right)^{3}$ the effective number of degrees of freedom associated to the sterile, and $m_{s}$ its real mass.

Table $\mathrm{V}$ depicts the $95 \%$ CL constraints on the active and sterile neutrino masses as well as on the total number of massive neutrinos $N_{\text {eff. Notice that the mean }}$ value of $N_{\text {eff }}$ is, in general, slightly larger than in the case in which the sterile neutrinos are considered as massless particles due to the fact that $m_{s}^{\text {eff }}$ and $N_{\text {eff }}$ are positively correlated. Indeed, there exists a physical lower prior for $N_{\text {eff }}$ of 3.046 which is not needed in the case of three active neutrinos plus extra massless species. We quote exclusively the 95\% CL upper limit for the cases in which the $95 \% \mathrm{CL}$ lower limit is set by the physical prior of 3.046. Concerning the bounds on the sum of the three active neutrinos, they are more stringent than in the massless sterile neutrino-like scenario because $\sum m_{\nu}$ and $m_{s}^{\text {eff }}$ are also positively correlated. As in the massless sterile neutrino-like analyses, larger values of $N_{\text {eff }}$ will be favoured by data when HST measurements are included. The addition of BBN bounds reduce the errors on $N_{\text {eff }}$ significantly, alleviating the degeneracies between $N_{\text {eff }}$ and the active/sterile neutrino masses. Table $\mathrm{V}$ contains the BBN constraints obtained using the fitting functions for the theoretical deuterium and helium primordial abundances from Ref. 68, which, as in the massless extra dark radiation case, are found to provide the most conservative bounds. We find $\sum m_{\nu}<0.27 \mathrm{eV}$, $m_{s}^{\text {eff }}<0.14 \mathrm{eV}$ and $N_{\text {eff }}=3.28_{-0.21}^{+0.22}$ at $95 \% \mathrm{CL}$ from the analysis of CMB data, BOSS DR11 BAO, additional BAO measurements, WiggleZ full-shape large scale structure information, the HST $H_{0}$ prior and BBN light elements abundances information with the most recent measurements of the primordial deuterium abundances from Ref. [52], indicating no significant preference for $N_{\text {eff }}>3$. However, when considering primordial deuterium measurements from Ref. 68, there exists a preference for $N_{\text {eff }}>3$ at the $3 \sigma$ level (mildly stronger when HST data is also considered in the analyses). This preference is similar to that found in the extra massless case, although notice that in this case there exists a lower prior on $N_{\text {eff }}=3.046$ and therefore the mean value of $N_{\text {eff }}$ will always be larger than its standard prediction. If we instead use the theoretical functions for the helium and deuterium abundances from Refs. [52, 72, we get similar conclusions to those found in the massless dark radiation case: a $3-4 \sigma$ preference for $N_{\text {eff }}>3$ is always 


\begin{tabular}{|c|c|c|c|c|c|c|c|c|}
\hline & CMB+DR11 & $\begin{array}{c}\text { CMB+DR11 } \\
+\mathrm{HST}\end{array}$ & $\begin{array}{c}\text { CMB+DR11 } \\
+ \text { WZ }\end{array}$ & $\begin{array}{c}\text { CMB+DR11 } \\
+\mathrm{WZ}+\mathrm{HST}\end{array}$ & $\begin{array}{c}\text { CMB+DR11 } \\
+\mathrm{WZ}+\mathrm{BAO}+\mathrm{HST}\end{array}$ & $\begin{array}{c}\text { CMB+DR11 } \\
+\mathrm{BAO}\end{array}$ & $\begin{array}{l}\text { CMB+DR11 } \\
+\mathrm{BAO}+\mathrm{HST}\end{array}$ & $\begin{array}{c}\text { CMB+DR11 } \\
+\mathrm{BAO+SNLS}\end{array}$ \\
\hline$\Sigma m_{\nu}[\mathrm{eV}]$ & $<0.28$ & $<0.27$ & $<0.28$ & $<0.30$ & $<0.31$ & $<0.30^{a}$ & $<0.29$ & $<0.26$ \\
\hline$m_{s}^{\mathrm{eff}}[\mathrm{eV}]$ & $<0.29$ & $<0.28$ & $<0.60$ & $<0.28$ & $<0.25$ & $<0.27^{a}$ & $<0.28$ & $<0.31$ \\
\hline$N_{\text {eff }}$ & $<4.01$ & $3.73_{-0.51}^{+0.51}$ & $<3.89$ & $<4.06$ & $3.64_{-0.48}^{+0.48}$ & $3.57_{-0.50}^{+0.50 a}$ & $<4.16$ & $<4.02$ \\
\hline \multicolumn{9}{|l|}{$\begin{array}{l}\text { SZ Clusters\& } \\
\text { CFHTLens }\end{array}$} \\
\hline$\Sigma m_{\nu}[\mathrm{eV}]$ & $<0.40$ & $<0.43$ & $<0.36$ & $<0.41$ & $<0.43$ & $<0.43$ & $<0.39$ & $<0.37$ \\
\hline$m_{s}^{\mathrm{eff}}[\mathrm{eV}]$ & $<0.50$ & $<0.48$ & $<1.37$ & $<0.39$ & $<0.34$ & $<0.49$ & $<0.59$ & $<0.59$ \\
\hline$N_{\text {eff }}$ & $<3.90$ & $3.67_{-0.55}^{+0.49}$ & $<3.77$ & $<4.08$ & $3.67_{-0.45}^{+0.51}$ & $3.47_{-0.39}^{+0.51}$ & $<4.01$ & $<3.85$ \\
\hline \multicolumn{9}{|l|}{ SZ Clusters } \\
\hline$\Sigma m_{\nu}[\mathrm{eV}]$ & $<0.40$ & $<0.42$ & $<0.36$ & $<0.41$ & $<0.42$ & $<0.41$ & $<0.39$ & $<0.38$ \\
\hline$m_{s}^{\mathrm{eff}}[\mathrm{eV}]$ & $<0.49$ & $<0.48$ & $<1.36$ & $<0.39$ & $<0.34$ & $<0.49$ & $<0.53$ & $<0.59$ \\
\hline$N_{\text {eff }}$ & $<3.90$ & $3.66_{-0.55}^{+0.49}$ & $<3.77$ & $<4.06$ & $3.66_{-0.45}^{+0.50}$ & $3.46_{-0.38}^{+0.41}$ & $<4.02$ & $<3.85$ \\
\hline \multicolumn{9}{|l|}{ CFHTLens } \\
\hline$\Sigma m_{\nu}[\mathrm{eV}]$ & $<0.35$ & $<0.33$ & $<0.32$ & $<0.35$ & $<0.35$ & $<0.39$ & $<0.34$ & $<0.31$ \\
\hline$m_{s}^{\mathrm{eff}}[\mathrm{eV}]$ & $<0.39$ & $<0.39$ & $<1.16$ & $<0.34$ & $<0.29$ & $<0.37$ & $<0.43$ & $<0.47$ \\
\hline$N_{\text {eff }}$ & $<3.94$ & $3.68_{-0.51}^{+0.51}$ & $<3.85$ & $<4.06$ & $3.63_{-0.49}^{+0.49}$ & $3.50_{-0.44}^{+0.48}$ & $<4.09$ & $<3.94$ \\
\hline \multicolumn{9}{|l|}{$\mathrm{BBN}$} \\
\hline$\Sigma m_{\nu}[\mathrm{eV}](D / H)_{p} 52$ & $<0.28$ & $<0.23$ & $<0.25$ & $<0.24$ & $<0.27$ & $<0.39$ & $<0.24$ & $<0.23$ \\
\hline$\Sigma m_{\nu}[\mathrm{eV}](D / H)_{p} 68$ & $<0.27$ & $<0.25$ & $<0.28$ & $<0.28$ & $<0.28$ & $<0.29$ & $<0.26$ & $<0.25$ \\
\hline$m_{s}^{\mathrm{eff}}[\mathrm{eV}](D / H)_{p} 52$ & $<0.45$ & $<0.34$ & $<0.37$ & $<0.46$ & $<0.14$ & $<0.24$ & $<0.56$ & $<0.62$ \\
\hline$m_{s}^{\mathrm{eff}}[\mathrm{eV}](D / H)_{p} 68$ & $<0.27$ & $<0.25$ & $<0.29$ & $<0.24$ & $<0.23$ & $<0.27$ & $<0.26$ & $<0.27$ \\
\hline$N_{\text {eff }}(D / H)_{p} 52$ & $<3.41$ & $<3.53$ & $<3.49$ & $<3.58$ & $3.28_{-0.21}^{+0.22}$ & $3.25_{-0.17}^{+0.17}$ & $<3.47$ & $<3.43$ \\
\hline$N_{\text {eff }}(D / H)_{p} \underline{68}$ & $3.48_{-0.35}^{+0.37}$ & $3.59_{-0.34}^{+0.35}$ & $3.45_{-0.38}^{+0.33}$ & $3.56_{-0.34}^{+0.34}$ & $3.56_{-0.32}^{+0.33}$ & $3.50_{-0.36}^{+0.35}$ & $3.59_{-0.45}^{+0.35}$ & $3.50_{-0.37}^{+0.36}$ \\
\hline
\end{tabular}

${ }^{a}$ These limits have been obtained by imposing an additional prior on the thermal velocity of sterile neutrinos. See discussion in the text for further details.

TABLE V: 95\% CL constraints on the active (sterile) neutrino masses, $\Sigma m_{\nu}$ ( $\left.m_{s}^{\text {eff }}\right)$, in eV, and on the total number of massive neutrino species, $N_{\text {eff }}$, from the different combinations of data sets explored here. When BBN bounds are included, the first (second) raw refers to the constraints obtained combining the primordial deuterium values from Ref. [52] (68]) $(D / H)_{p}=(2.53 \pm 0.04) \times 10^{-5}\left((D / H)_{p}=(2.87 \pm 0.22) \times 10^{-5}\right)$ with measurements of the helium mass fraction $Y_{p}=0.254 \pm 0.003$ from Ref. 53. 

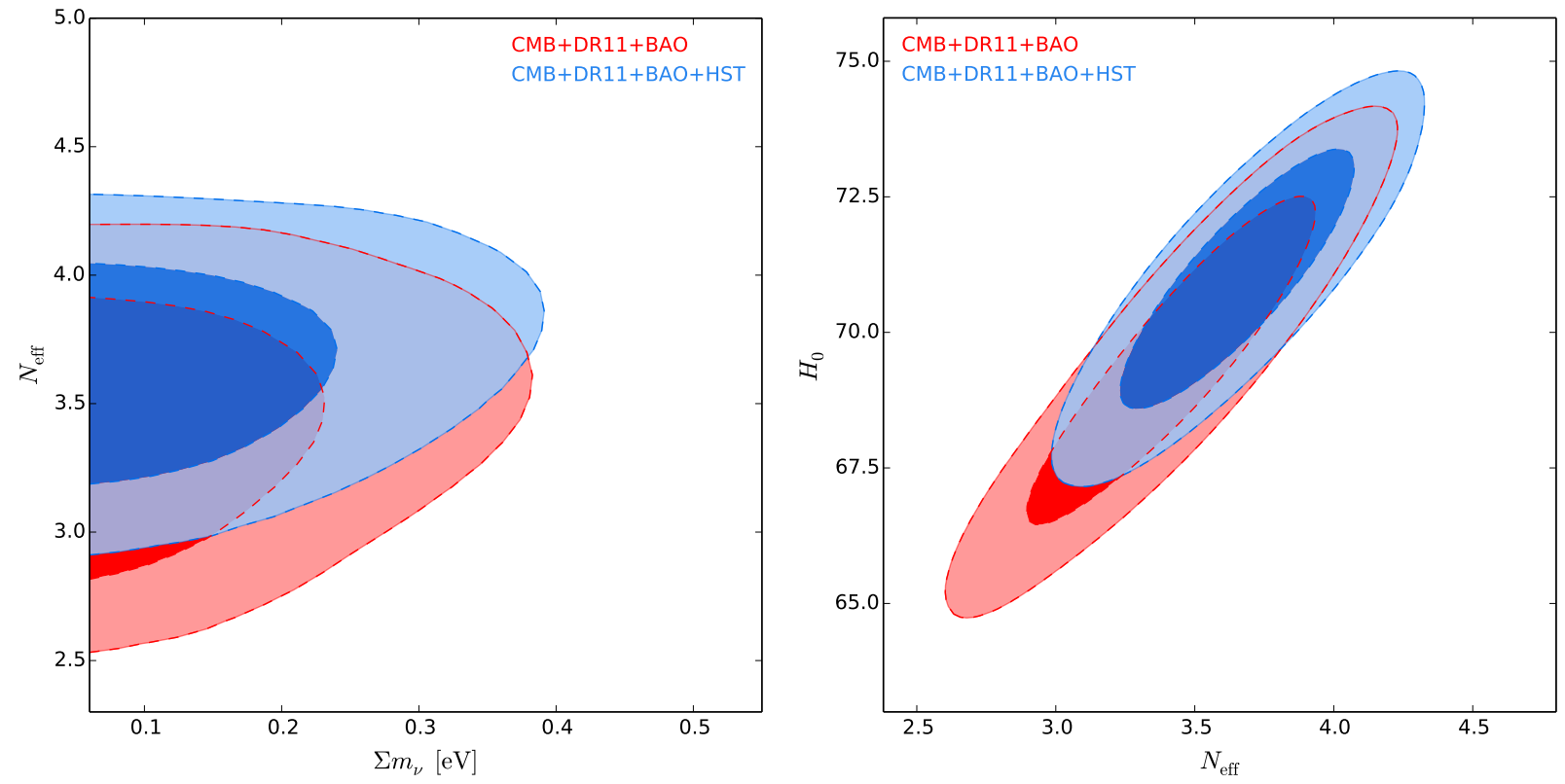

FIG. 3: Left panel: the red contours show the $68 \%$ and $95 \%$ CL allowed regions from the combination of CMB data, BOSS DR11 BAO measurements and additional BAO measurements in the $\left(\sum m_{\nu}(\mathrm{eV}), N_{\text {eff }}\right)$ plane. The blue contours depict the constraints after a prior on the Hubble constant from HST is added in the analysis. Right panel: as in the left panel but in the $\left(N_{\text {eff }}, H_{0}\right)$ plane. 
present. The bounds on the neutrino masses are, as in the massless case, mildly loosened. The constraints quoted above translate into $\sum m_{\nu}<0.28 \mathrm{eV}, m_{s}^{\text {eff }}<0.22 \mathrm{eV}$ and $N_{\text {eff }}=3.50_{-0.28}^{+0.27}\left(\sum m_{\nu}<0.30 \mathrm{eV}, m_{s}^{\mathrm{eff}}<0.24 \mathrm{eV}\right.$ and $\left.N_{\text {eff }}=3.64_{-0.33}^{+0.33}\right)$ at $95 \% \mathrm{CL}$ from the analysis of CMB data, BOSS DR11 BAO, additional BAO measurements, WiggleZ full-shape large scale structure information, the HST $H_{0}$ prior and BBN light elements abundances information with the most recent measurements of the primordial deuterium abundances from Ref. [52] (68]).

We have also found that the posterior distribution obtained from the $\mathrm{CMB}+\mathrm{DR} 11+\mathrm{BAO}$ dataset (without the addition of any $\mathrm{BBN}$ or $\sigma_{8}$ information) is multimodal. In fact, we find that the probability density is significantly different from zero, other than for $m_{\text {eff }} \lesssim 0.3$ $\mathrm{eV}$ (as for the other datasates), also for $m_{\mathrm{eff}} \gtrsim 1 \mathrm{eV}$. A further inspection of the chains has shown that these two regions roughly corresponds to the two cases of a hot/warm (at recombination) sterile neutrino, with a mass-to-temperature ratio at that time $m_{s} / T_{s \text {,rec }} \lesssim 10$, and of a cold sterile with $m_{s} / T_{s, \text { rec }} \gtrsim 100$. The limits quoted in Tab. $\mathrm{V}$ for the $\mathrm{CMB}+\mathrm{DR} 11+\mathrm{BAO}$ dataset, in the basic case where no other information is considered, have been obtained by postprocessing the chains in order to keep only those models with $m_{s} / T_{s \text {,rec }} \lesssim 10$. This is consistent with the purpose of the paper of costraining the presence of a hot component in addition to active neutrinos. We have also verified that these limits are reasonably stable with respect to the choice of the value of the mass-to-temperature ratio at which to cut the distribution, as long as this value lies inside the lowprobability region $10 \lesssim m_{s} / T_{s, \text { rec }} \lesssim 100$. It still has to be clarified which, if any in particular, of the BAO datasets is responsible for the appearance of the "cold sterile" region in the posterior probability, and to which feature in the data this is possibly related. A very preliminar analysis, performed using only one at a time among the DR7, $6 \mathrm{dF}$ and WiggleZ BAO datasets, seems to show that this effect is mainly driven by the first two datasets, while using only the WiggleZ BAO measurements yields naturally an upper limit for $m_{\text {eff }}$ of about $0.3 \mathrm{eV}$, without any need to exclude a priori the cold region. However a more robust and precise assessment of the role of the different datasets would certainly require a more detailed analysis that goes beyond the scope of the present paper.

Contrarily to the massless dark radiation case (and similarly to the thermal axion scenario), the addition of the constraints on the $\sigma_{8}$ and $\Omega_{m}$ cosmological parameters from the Planck-SZ cluster catalog on galaxy number counts does not lead to a non zero value for the neutrino masses. However, the bounds on the neutrino masses are less stringent when adding the Planck-SZ or the CFHTLens constraints on the $\sigma_{8}$ and $\Omega_{m}$ cosmological parameters, due to the lower $\sigma_{8}$ preferred by the former data sets, which favours higher values for the thermal relic masses. After considering the inclusion of Planck SZ clusters and CFHTLens information to CMB data, BOSS DR11 BAO and additional BAO measurements and the HST $H_{0}$ prior, the $95 \%$ CL bounds on the active and the sterile neutrino parameters are $\sum m_{\nu}<0.39 \mathrm{eV}$, $m_{s}^{\text {eff }}<0.59 \mathrm{eV}$ and $N_{\text {eff }}<4.01$.

The bounds quoted in Tab. V have been obtained using the BBN theoretical prediction for helium in the $\mathrm{CMB}$ data analysis, as in the case of extra massless species. We have also performed in this massive case the exercise of fixing the helium fraction $Y_{p}$ in the Monte Carlo Markov Chain analyses of CMB data and assume that $Y_{p}$ is an independent parameter constrained by BBN observations only. Again, as in the massless case, we find larger values for the mean value of $N_{\text {eff }}$ (and, consequently, slightly larger bounds on both the active and sterile neutrino masses) when neglecting the BBN consistency relation in the MCMC analyses.

Figure 4, left panel, shows the degeneracy between the $\sum m_{\nu}$ and the total number of neutrino species $N_{\text {eff }}$ (which accounts for the contribution of the three active neutrino species plus $\Delta N_{\text {eff }}$ massive sterile neutrinos). The red contours depict the $68 \%$ and $95 \% \mathrm{CL}$ allowed regions resulting from the combination of CMB, BOSS DR11 BAO measurements, and full shape power spectrum measurements from the WiggleZ survey. Notice that the allowed values of $N_{\text {eff }}$ are slightly larger than in the massless dark radiation scenario, since sub-eV massive sterile neutrinos are contributing to the matter energy density at the recombination period and therefore a larger value of $N_{\text {eff }}$ will be required to leave unchanged both the angular location and the height of the first acoustic peak. The blue region depicts the results considering both the HST $H_{0}$ prior and the remaining BAO data as well in the analysis. The right panel of Fig. 4, illustrates the degeneracy between the active and the sterile neutrino masses, since both active and sterile sub-eV massive neutrinos contribute to the matter energy density at decoupling, and both are free streaming relics which suppress structure formation at small scales, after they become non relativistic.

\section{CMB CONSTRAINTS INCLUDING THE RECENT RESULTS FROM THE BICEP2 EXPERIMENT}

Very recently, the BICEP2 experiment 74 claimed a detection at about $5.9 \sigma$ for B-mode polarization on large angular scales, compatible with the presence of a tensor component with amplitude $r_{0.002}=0.2_{-0.05}^{+0.06}$ at $68 \%$ c.l.. It is therefore interesting to evaluate the impact of this measurement for the effective number of relativistic species and neutrino masses. We have therefore performed an analysis including a tensor component (with zero running). The results are presented in Fig. 5. As we can see, when the BICEP2 data are included, an extra background of relativistic particle is preferred with $N_{\text {eff }}=4.00 \pm 0.41$ at $68 \%$ c.l.. CMB data alone is therefore suggesting a value for $N_{\text {eff }}>3$ at good significance. This result comes from the apparent tension between the 

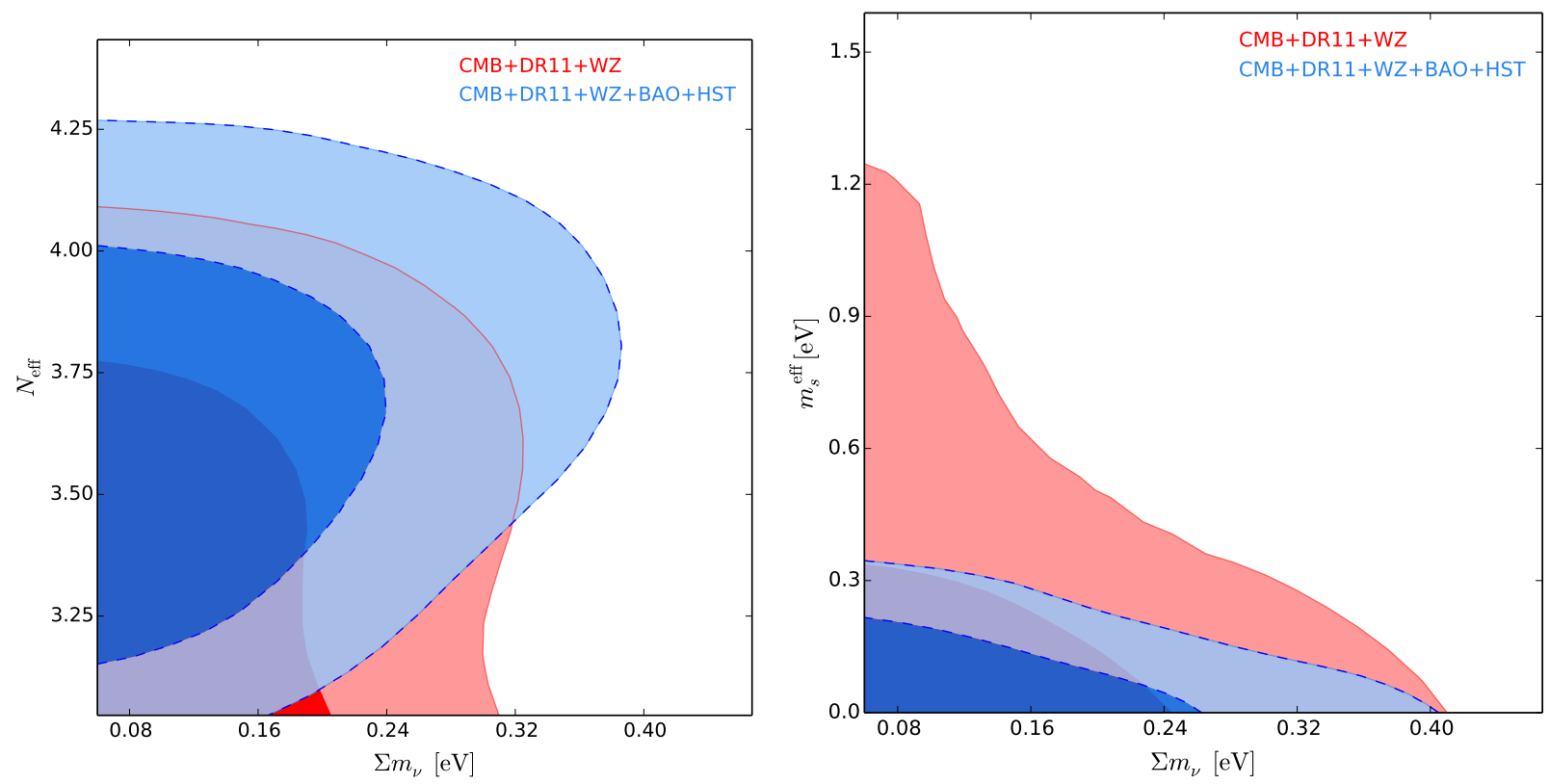

FIG. 4: Left panel: the red contours show the $68 \%$ and $95 \%$ CL allowed regions from the combination of CMB data, BOSS DR11 BAO measurements and WiggleZ full shape power spectrum measurements in the $\left(\sum m_{\nu}(\mathrm{eV}), N_{\text {eff }}\right)$ plane. The blue contours depict the constraints after a prior on the Hubble constant from HST and the remaining BAO data are added in the analysis. Right panel: as in the left panel but in the $\left(\sum m_{\nu}(\mathrm{eV}), m_{s}^{\text {eff }}(\mathrm{eV})\right)$ plane.
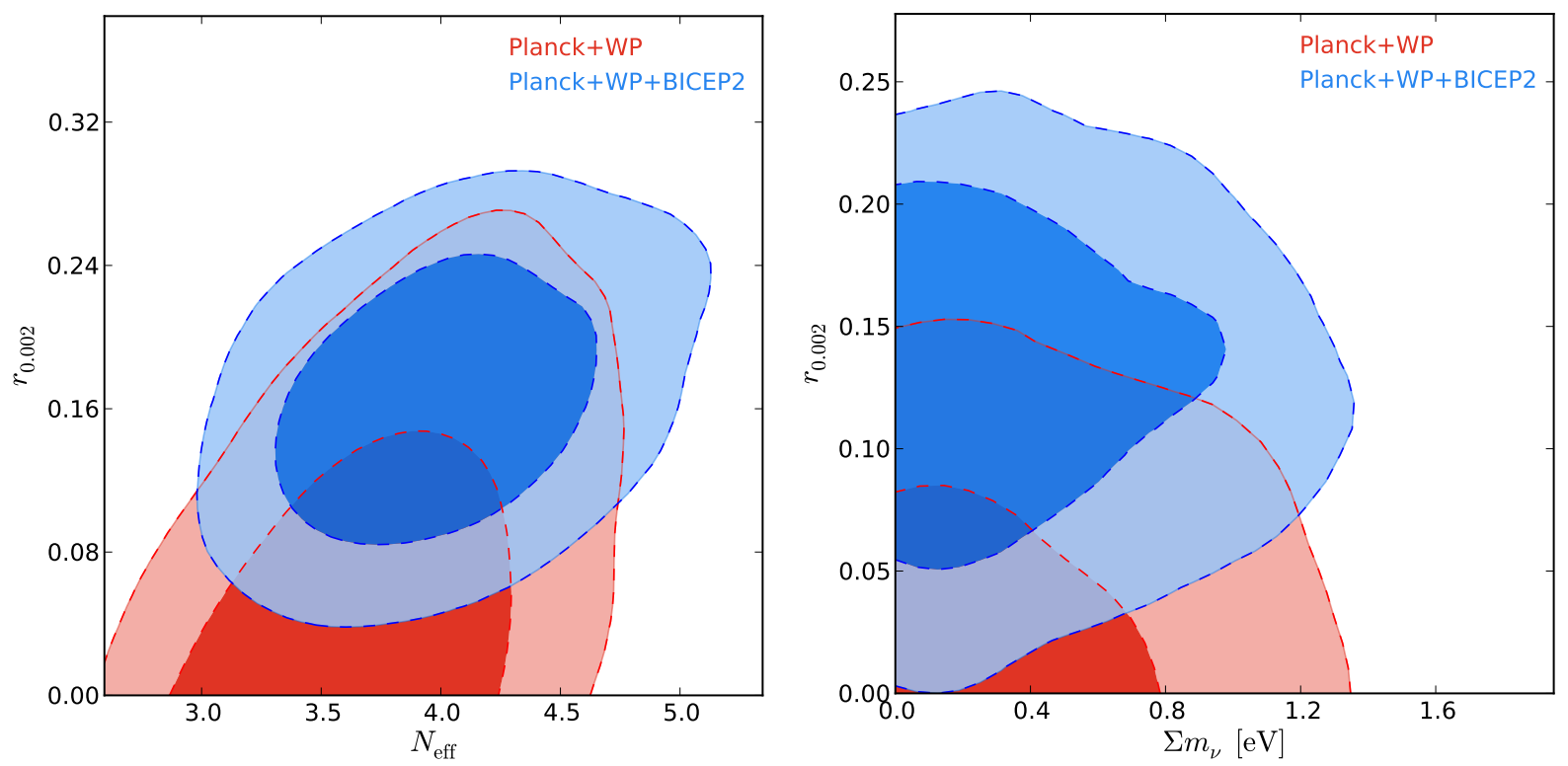

FIG. 5: Left panel: Constraints in the $N_{\text {eff }}$ vs $r$ plane from Planck+WP and Planck+WP+BICEP2 data. Notice how the inclusion of the BICEP2 constraint shifts the contours towards $N_{\text {eff }}>3$. Right panel: constraints on the $\Sigma m_{\nu}$ vs $r$ plane from Planck+WP and Planck+WP+BICEP2 data. In this case there is no indication for neutrino masses from the combination of CMB data.

Planck+WP limit of $r<0.11$ at $95 \%$ c.l. and the recent BICEP2 result. This tension appears as less evident when extra relativistic particles are included. We imagine a further preference for $N_{\text {eff }}>3$ if the HST data is included. The BICEP2 result does not affect the current constraints on neutrino masses as we can see from the right side of figure Fig. 5 . 


\section{CONCLUSIONS}

Standard cosmology includes hot thermal relics which refer to the three light, active neutrino flavours of the Standard Model of elementary particles. The largest effect of neutrino masses on the different cosmological observables arises from their free streamig nature: the non-relativistic neutrino overdensities will contribute to clustering only at scales larger than their free streaming scale, suppressing the growth of matter density fluctuations at small scales. CMB measurements from the Planck satellite, including the lensing likelihood, low- $\ell$ polarization measurements from WMAP 9-year data and Baryon Acoustic Oscillation (BAO) measurements from a number of surveys lead to the bound $\sum m_{\nu}<0.26 \mathrm{eV}$ at $95 \%$ CL.

However, the existence of extra hot relic components, as dark radiation relics, sterile neutrino species and/or thermal axions will change the cosmological neutrino mass constraints. Dark radiation (i.e. purely massless species) may arise in several extentions of the Standard Model of elementary particles, as, for instance, in asymmetric dark matter models. On the other hand, the existence of extra massive species is well motivated by either the so-called neutrino oscillation anomalies (in the case of sterile neutrino species) or by the strong CP problem (in the case of thermal axions). Both extra, sterile neutrino species and axions have an associated free streaming scale, reducing the growth of matter fluctuations at small scales. These extra species will also contribute to the effective number of relativistic degrees of freedom $N_{\text {eff }}$, being $N_{\text {eff }}=3.046$ the standard value, corresponding to the three active neutrino contribution. The existence of extra light species at the Big Bang Nucleosynthesis (BBN) epoch modifies the light element abundances, especially the primordial helium mass fraction.

We have presented here the constraints on the masses of the different thermal relics in different scenarios using the available cosmological data in the beginning of this year 2014. The data combination used here includes also the recent and most precise distance BAO constraints to date from the BOSS Data Release 11 (DR11) results [54, see also Refs. 55 57. The tightest limit we find in the minimal three active massive neutrino scenario is $\sum m_{\nu}<0.22 \mathrm{eV}$ at $95 \% \mathrm{CL}$ from the combination of CMB data, BAO data and HST measurements of the Hubble constant. The addition of the constraints on $\sigma_{8}$ and $\Omega_{m}$ from the CFHTLens survey displaces the bounds on the neutrino mass to higher values. However, the constraint on $\sigma_{8}$ and $\Omega_{m}$ from the Planck-SZ cluster catalog on galaxy number counts favours a non zero value for the sum of the three active neutrino masses of $\sim 0.3 \mathrm{eV}$ at $4 \sigma$, see also Refs. [48, 49].

When considering simultaneously thermal axions and active massive neutrino species, and including CMB, BOSS BAO DR11, additional BAO measurements, WiggleZ power spectrum (full shape) information, the $H_{0}$ HST prior and BBN light element abundances, the
95\% CL bounds are $\sum m_{\nu}<0.25 \mathrm{eV}$ and $m_{a}<0.57 \mathrm{eV}$ $\left(\sum m_{\nu}<0.21 \mathrm{eV}\right.$ and $\left.m_{a}<0.61 \mathrm{eV}\right)$ using recent (previous) deuterium estimates from [52 (68) and helium constraints from Ref. [53].

Neither the addition of weak lensing constraints on the $\sigma_{8}-\Omega_{m}$ relationship from the CFHTLens experiment nor from the Planck SZ cluster number counts favours nonzero thermal relic masses, except for few cases in which the Planck SZ cluster number counts information is considered together with the HST $H_{0}$ prior (or SNIa luminosity distances) and all the BAO measurements. Only in this case there exists a mild $\sim 2.2 \sigma$ preference for a non zero axion mass of $0.6 \mathrm{eV}$. Concerning neutrino masses, there exists evidence for a neutrino mass of $\sim 0.2 \mathrm{eV}$ at the $\sim 3 \sigma$ level exclusively for the case in which CMB data is combined with BOSS BAO DR11 measurements and full-shape power spectrum information from the WiggleZ galaxy survey.

In the case in which we consider both massive neutrinos and $\Delta N_{\text {eff }}$ dark radiation species, the neutrino mass bounds are less stringent than in standard three neutrino massive case due to the large degeneracy between $\sum m_{\nu}$ and $N_{\text {eff }}$, finding $\sum m_{\nu}<0.31 \mathrm{eV}$ and $N_{\text {eff }}=3.45_{-0.54}^{+0.59}$ at 95\% CL from the combination of CMB data and BOSS DR11 BAO measurements. Contrarily to the massless dark radiation case, but similarly to the thermal axion scenario, the addition of the constraints on the $\sigma_{8}$ and $\Omega_{m}$ cosmological parameters from the Planck-SZ cluster catalog on galaxy number counts does not lead to a non zero value for the neutrino masses. After considering the inclusion of Planck SZ clusters and CFHTLens information to CMB data, BOSS DR11 BAO, additional BAO measurements and the HST $H_{0}$ prior, the $95 \% \mathrm{CL}$ bounds on the active and the sterile neutrino parameters are $\sum m_{\nu}<0.39 \mathrm{eV}, m_{s}^{\mathrm{eff}}<0.59 \mathrm{eV}$ and $N_{\text {eff }}<4.01$.

Big Bang Nucleosynthesis constraints reduce both the mean value and the errors of $N_{\text {eff }}$ significantly. After the addition of the most recent measurements of deuterium 52 and helium 53, and using the theoretically derived fitting functions of Ref. 68, we find $\sum m_{\nu}<$ $0.24 \mathrm{eV}$ and $N_{\text {eff }}=3.25_{-0.24}^{+0.25}$ at $95 \%$ CL from the analysis of CMB data, WiggleZ power spectrum measurements and the HST $H_{0}$ prior finding no evidence for $N_{\text {eff }}>3$. If previous estimates of the deuterium primordial aundances are used in the analysis 68, there exists a $4(2.5) \sigma$ preference for $N_{\text {eff }}>3$, with (without) HST data included in the numerical analyses. If the additional sterile neutrino states are considered as massive species, a $\sim 3.5 \sigma$ preference for $N_{\text {eff }}>3$ still appears when considering BBN measurements (with previous estimates of the deuterium abundances from Ref. 68]) and the HST prior on the Hubble constant. The $2.5-4 \sigma$ preference for $N_{\text {eff }}>3$ always appears for both the massless and the massive extra hot relic scenarios when considering the theoretical fitting functions of Refs. [52, 72], independently of the deuterium measurements used in the analyses. Accurate measurements as well as sharp theoretical predictions of the primordial deuterium and 
helium light element abundances are therefore crucial to constrain the value of $N_{\text {eff. }}$.

Finally, we have considered the recent B-mode polarization measurements made by the BICEP2 experiment. Assuming that this detection is produced by a primordial tensor component, we have found that in a LCDM $+r$ scenario the presence of extra relativistic particles is significantly suggested by current Planck+WP+BICEP2 data with $N_{e f f}=4.00 \pm 0.41$ at $68 \%$ c.l.. An extra relativistic component therefore solves the current tension between the Planck and BICEP2 experiments on the amplitude of tensor modes.

\section{ACKNOWLEDGMENTS}

M.L. is supported by Ministero dell'Istruzione, dell'Università e della Ricerca (MIUR) through the PRIN grant Galactic and extragalactic polarized microwave emission' (contract number PRIN 2009XZ54H2-002). Most of this work was carried out while M.L. was visiting the Instituto de Física Corpuscular, whose hospitality is kindly acknowledged, supported by the grant Giovani ricercatori of the University of Ferrara, financed through the funds Fondi 5x1000 Anno 2010 and Fondi Unicredit 2013. O.M. is supported by the Consolider Ingenio project CSD2007-00060, by PROMETEO/2009/116, by the Spanish Ministry Science project FPA2011-29678 and by the ITN Invisibles PITN-GA-2011-289442.

\section{APPENDIX}

For axion thermalization purposes, only the axion-pion interaction will be relevant. To compute the axion decoupling temperature $T_{D}$ we follow the usual freeze out condition

$$
\Gamma\left(T_{D}\right)=H\left(T_{D}\right)
$$

The average rate $\pi+\pi \rightarrow \pi+a$ is given by [73]

$$
\Gamma=\frac{3}{1024 \pi^{5}} \frac{1}{f_{a}^{2} f_{\pi}^{2}} C_{a \pi}^{2} I,
$$

where

$$
C_{a \pi}=\frac{1-R}{3(1+R)}
$$

is the axion-pion coupling constant [73, and

$$
\begin{aligned}
I & =n_{a}^{-1} T^{8} \int d x_{1} d x_{2} \frac{x_{1}^{2} x_{2}^{2}}{y_{1} y_{2}} f\left(y_{1}\right) f\left(y_{2}\right) \\
& \times \int_{-1}^{1} d \omega \frac{\left(s-m_{\pi}^{2}\right)^{3}\left(5 s-2 m_{\pi}^{2}\right)}{s^{2} T^{4}}
\end{aligned}
$$

where $n_{a}=\left(\zeta_{3} / \pi^{2}\right) T^{3}$ is the number density for axions in thermal equilibrium, $f(y)=1 /\left(e^{y}-1\right)$ denotes the pion distribution function, $x_{i}=\left|\vec{p}_{i}\right| / T, y_{i}=E_{i} / T(i=1,2)$, $s=2\left(m_{\pi}^{2}+T^{2}\left(y_{1} y_{2}-x_{1} x_{2} \omega\right)\right)$, and we assume a common mass for the charged and neutral pions, $m_{\pi}=138 \mathrm{MeV}$.

We have numerically solved the freeze out equation Eq. (7), obtaining the axion decoupling temperature $T_{D}$ versus the axion mass $m_{a}$ (or, equivalently, versus the axion decay constant $f_{a}$ ). From the axion decoupling temperature, we can compute the current axion number density, related to the present photon density $n_{\gamma}=410.5 \pm 0.5 \mathrm{~cm}^{-3}$ via

$$
n_{a}=\frac{g_{\star S}\left(T_{0}\right)}{g_{\star S}\left(T_{D}\right)} \times \frac{n_{\gamma}}{2},
$$

where $g_{\star S}$ refers to the number of entropic degrees of freedom. At the current temperature, $g_{\star S}\left(T_{0}\right)=3.91$.
[1] J. Lesgourgues and S. Pastor, Adv. High Energy Phys. 2012, 608515 (2012) arXiv:1212.6154 [hep-ph]].

[2] J. Lesgourgues and S. Pastor, Phys. Rept. 429, 307 (2006) astro-ph/0603494.

[3] P. A. R. Ade et al. [Planck Collaboration], arXiv:1303.5076 [astro-ph.CO].

[4] J. Lesgourgues, L. Perotto, S. Pastor and M. Piat, Phys. Rev. D 73, 045021 (2006) astro-ph/0511735.

[5] B. A. Reid, L. Verde, R. Jimenez and O. Mena, JCAP 1001, 003 (2010) arXiv:0910.0008 [astro-ph.CO]].

[6] J. Hamann, S. Hannestad, J. Lesgourgues, C. Rampf and Y. Y. Y. Wong, JCAP 1007, 022 (2010) arXiv:1003.3999 [astro-ph.CO]].

[7] R. de Putter, O. Mena, E. Giusarma, S. Ho, A. Cuesta, H. -J. Seo, A. J. Ross and M. White et al., Astrophys. J. 761, 12 (2012) arXiv:1201.1909 [astro-ph.CO]].

[8] E. Giusarma, R. de Putter and O. Mena, Phys. Rev. D
87, no. 4, 043515 (2013) arXiv:1211.2154 [astro-ph.CO]].

[9] G. -B. Zhao, S. Saito, W. J. Percival, A. J. Ross, F. Montesano, M. Viel, D. P. Schneider and M. Manera et al., arXiv:1211.3741 [astro-ph.CO].

[10] G. Hinshaw, D. Larson, E. Komatsu, D. N. Spergel, C. L. Bennett, J. Dunkley, M. R. Nolta and M. Halpern et al., arXiv:1212.5226 [astro-ph.CO].

[11] Z. Hou, C. L. Reichardt, K. T. Story, B. Follin, R. Keisler, K. A. Aird, B. A. Benson and L. E. Bleem et al., arXiv:1212.6267 [astro-ph.CO].

[12] J. L. Sievers, R. A. Hlozek, M. R. Nolta, V. Acquaviva, G. E. Addison, P. A. R. Ade, P. Aguirre and M. Amiri et al., arXiv:1301.0824 [astro-ph.CO].

[13] M. Archidiacono, E. Giusarma, A. Melchiorri and O. Mena, Phys. Rev. D 87, 103519 (2013) arXiv:1303.0143 [astro-ph.CO]].

[14] E. Giusarma, R. de Putter, S. Ho and O. Mena, 
Phys. Rev. D 88, 063515 (2013) arXiv:1306.5544 [astroph.CO]].

[15] M. Archidiacono, E. Giusarma, S. Hannestad and O. Mena, arXiv:1307.0637 [astro-ph.CO].

[16] S. Riemer-Srensen, D. Parkinson and T. M. Davis, arXiv:1306.4153 [astro-ph.CO].

[17] J. -W. Hu, R. -G. Cai, Z. -K. Guo and B. Hu, arXiv:1401.0717 [astro-ph.CO].

[18] C. L. Bennett, D. Larson, J. L. Weiland, N. Jarosik, G. Hinshaw, N. Odegard, K. M. Smith and R. S. Hill et al., arXiv:1212.5225 [astro-ph.CO].

[19] A. G. Riess, L. Macri, S. Casertano, H. Lampeitl, H. C. Ferguson, A. V. Filippenko, S. W. Jha and W. Li et al., Astrophys. J. 730, 119 (2011) [Erratum-ibid. 732, 129 (2011)] arXiv:1103.2976 [astro-ph.CO]].

[20] W. J. Percival et al. [SDSS Collaboration], Mon. Not. Roy. Astron. Soc. 401, 2148 (2010) arXiv:0907.1660 [astro-ph.CO]].

[21] N. Padmanabhan, X. Xu, D. J. Eisenstein, R. Scalzo, A. J. Cuesta, K. T. Mehta and E. Kazin, arXiv:1202.0090 [astro-ph.CO].

[22] C. Blake, E. Kazin, F. Beutler, T. Davis, D. Parkinson, S. Brough, M. Colless and C. Contreras et al., Mon. Not. Roy. Astron. Soc. 418, 1707 (2011) arXiv:1108.2635 [astro-ph.CO]].

[23] K. S. Dawson et al. [BOSS Collaboration], arXiv:1208.0022 [astro-ph.CO].

[24] D. J. Eisenstein et al. [SDSS Collaboration], Astron. J. 142, 72 (2011) arXiv:1101.1529 [astro-ph.IM]].

[25] L. Anderson, E. Aubourg, S. Bailey, D. Bizyaev, M. Blanton, A. S. Bolton, J. Brinkmann and J. R. Brownstein et al., Mon. Not. Roy. Astron. Soc. 427, no. 4, 3435 (2013) arXiv:1203.6594 [astro-ph.CO]].

[26] F. Beutler, C. Blake, M. Colless, D. H. Jones, L. StaveleySmith, L. Campbell, Q. Parker and W. Saunders et al., Mon. Not. Roy. Astron. Soc. 416, 3017 (2011) arXiv:1106.3366 [astro-ph.CO]].

[27] R. de Putter, E. V. Linder and A. Mishra, arXiv:1401.7022 [astro-ph.CO].

[28] J. Hamann, S. Hannestad, G. G. Raffelt, I. Tamborra and Y. Y. Y. Wong, Phys. Rev. Lett. 105, 181301 (2010) arXiv:1006.5276 [hep-ph]].

[29] E. Giusarma, M. Corsi, M. Archidiacono, R. de Putter, A. Melchiorri, O. Mena and S. Pandolfi, Phys. Rev. D 83, 115023 (2011) arXiv:1102.4774 [astro-ph.CO]].

[30] E. Giusarma, M. Archidiacono, R. de Putter, A. Melchiorri and O. Mena, Phys. Rev. D 85, 083522 (2012) arXiv:1112.4661 [astro-ph.CO]].

[31] J. Hamann, S. Hannestad, G. G. Raffelt and Y. Y. Y. Wong, JCAP 1109, 034 (2011) arXiv:1108.4136 [astro-ph.CO]].

[32] A. Melchiorri, O. Mena and A. Slosar, Phys. Rev. D 76, 041303 (2007) arXiv:0705.2695 [astro-ph]].

[33] S. Hannestad, A. Mirizzi, G. G. Raffelt and Y. Y. Y. Wong, JCAP 0708, 015 (2007) arXiv:0706.4198 [astro-ph]].

[34] S. Hannestad, A. Mirizzi, G. G. Raffelt and Y. Y. Y. Wong, JCAP 0804, 019 (2008) arXiv:0803.1585 [astro-ph]].

[35] S. Hannestad, A. Mirizzi, G. G. Raffelt and Y. Y. Y. Wong, JCAP 1008, 001 (2010) arXiv:1004.0695 [astro-ph.CO]].

[36] M. Archidiacono, S. Hannestad, A. Mirizzi, G. Raffelt and Y. Y. Y. Wong, JCAP 1310, 020 (2013)
arXiv:1307.0615 [astro-ph.CO]].

[37] M. Blennow, E. Fernandez-Martinez, O. Mena, J. Redondo and P. Serra, JCAP 1207, 022 (2012) arXiv:1203.5803 [hep-ph]].

[38] R. Diamanti, E. Giusarma, O. Mena, M. Archidiacono and A. Melchiorri, Phys. Rev. D 87, no. 6, 063509 (2013) arXiv:1212.6007 [astro-ph.CO]].

[39] U. Franca, R. A. Lineros, J. Palacio and S. Pastor, Phys. Rev. D 87, 123521 (2013) arXiv:1303.1776 [astroph.CO]].

[40] K. N. Abazajian, M. A. Acero, S. K. Agarwalla, A. A. Aguilar-Arevalo, C. H. Albright, S. Antusch, C. A. Arguelles and A. B. Balantekin et al., arXiv:1204.5379 [hep-ph].

[41] J. Kopp, P. A. N. Machado, M. Maltoni and T. Schwetz, JHEP 1305, 050 (2013) arXiv:1303.3011 [hep-ph]].

[42] A. Melchiorri, O. Mena, S. Palomares-Ruiz, S. Pascoli, A. Slosar and M. Sorel, JCAP 0901, 036 (2009) arXiv:0810.5133 [hep-ph]].

[43] M. Archidiacono, N. Fornengo, C. Giunti and A. Melchiorri, Phys. Rev. D 86, 065028 (2012) arXiv:1207.6515 [astro-ph.CO]].

[44] M. Archidiacono, N. Fornengo, C. Giunti, S. Hannestad and A. Melchiorri, arXiv:1302.6720 [astro-ph.CO].

[45] A. Mirizzi, G. Mangano, N. Saviano, E. Borriello, C. Giunti, G. Miele and O. Pisanti, Phys. Lett. B 726, 8 (2013) arXiv:1303.5368 [astro-ph.CO]].

[46] E. Di Valentino, A. Melchiorri and O. Mena, JCAP 1311, 018 (2013) arXiv:1304.5981.

[47] R. D. Peccei and H. R. Quinn, Phys. Rev. Lett. 38, 1440 (1977); R. D. Peccei and H. R. Quinn, Phys. Rev. D 16, 1791 (1977).

[48] J. Hamann and J. Hasenkamp, JCAP 1310, 044 (2013) arXiv:1308.3255 [astro-ph.CO]].

[49] M. Wyman, D. H. Rudd, R. A. Vanderveld and W. Hu, arXiv:1307.7715 [astro-ph.CO].

[50] P. A. R. Ade et al. [Planck Collaboration], arXiv:1303.5080 [astro-ph.CO].

[51] C. Heymans, E. Grocutt, A. Heavens, M. Kilbinger, T. D. Kitching, F. Simpson, J. Benjamin and T. Erben et al., arXiv:1303.1808 [astro-ph.CO].

[52] R. Cooke, M. Pettini, R. A. Jorgenson, M. T. Murphy and C. C. Steidel, arXiv:1308.3240 [astro-ph.CO].

[53] Y. I. Izotov, G. Stasinska and N. G. Guseva, arXiv:1308.2100 [astro-ph.CO].

[54] L. Anderson, E. Aubourg, S. Bailey, F. Beutler, V. Bhardwaj, M. Blanton, A. S. Bolton and J. Brinkmann et al., arXiv:1312.4877 [astro-ph.CO].

[55] L. Samushia, B. A. Reid, M. White, W. J. Percival, A. J. Cuesta, G. -B. Zhao, A. J. Ross and M. Manera et al., arXiv:1312.4899 [astro-ph.CO].

[56] A. G. Sanchez, F. Montesano, E. A. Kazin, E. Aubourg, F. Beutler, J. Brinkmann, J. R. Brownstein and A. J. Cuesta et al., arXiv:1312.4854 [astro-ph.CO].

[57] C. -H. Chuang, F. Prada, F. Beutler, D. J. Eisenstein, S. Escoffier, S. Ho, J. -P. Kneib and M. Manera et al., arXiv:1312.4889 [astro-ph.CO].

[58] A. Lewis, A. Challinor and A. Lasenby, Astrophys. J. 538, 473 (2000) arXiv:astro-ph/9911177.

[59] A. Lewis and S. Bridle, Phys. Rev. D 66, 103511 (2002) arXiv:astro-ph/0205436.

[60] P. A. R. Ade et al. [Planck Collaboration], arXiv:1303.5062 [astro-ph.CO].

[61] P. A. R. Ade et al. [Planck Collaboration], 
arXiv:1303.5075 [astro-ph.CO].

[62] P. A. R. Ade et al. [Planck Collaboration], arXiv:1303.5077 [astro-ph.CO].

[63] S. Das, T. Louis, M. R. Nolta, G. E. Addison, E. S. Battistelli, J R. Bond, E. Calabrese and D. C. M. J. Devlin et al., arXiv:1301.1037 [astro-ph.CO].

[64] C. L. Reichardt, L. Shaw, O. Zahn, K. A. Aird, B. A. Benson, L. E. Bleem, J. E. Carlstrom and C. L. Chang et al., Astrophys. J. 755, 70 (2012) arXiv:1111.0932 [astroph.CO]].

[65] D. J. Eisenstein and W. Hu, Astrophys. J. 496, 605 (1998) astro-ph/9709112.

[66] D. Parkinson, S. Riemer-Sorensen, C. Blake, G. B. Poole, T. M. Davis, S. Brough, M. Colless and C. Contreras et al., Phys. Rev. D 86, 103518 (2012) arXiv:1210.2130 [astro-ph.CO]].

[67] A. Conley, J. Guy, M. Sullivan, N. Regnault, P. Astier, C. Balland, S. Basa and R. G. Carlberg et al., Astrophys.
J. Suppl. 192, 1 (2011) arXiv:1104.1443 [astro-ph.CO]].

[68] F. Iocco, G. Mangano, G. Miele, O. Pisanti and P. D. Serpico, Phys. Rept. 472, 1 (2009) arXiv:0809.0631 [astro$\mathrm{ph}]$.

[69] O. Pisanti, A. Cirillo, S. Esposito, F. Iocco, G. Mangano, G. Miele and P. D. Serpico, Comput. Phys. Commun. 178, 956 (2008) arXiv:0705.0290 [astro-ph]].

[70] A. Arbey, Comput. Phys. Commun. 183, 1822 (2012) arXiv:1106.1363 [astro-ph.CO]].

[71] Z. Hou, R. Keisler, L. Knox, M. Millea and C. Reichardt, Phys. Rev. D 87, 083008 (2013) arXiv:1104.2333 [astroph.CO]].

[72] G. Steigman, Adv. High Energy Phys. 2012, 268321 (2012) arXiv:1208.0032 [hep-ph]].

[73] S. Chang and K. Choi, Phys. Lett. B 316, 51 (1993)

[74] P. A. R. Ade et al. [BICEP2 Collaboration], arXiv:1403.3985 [astro-ph.CO]. 